\title{
Bayesian estimation of spatial filters with Morans eigenvectors and hierarchical shrinkage priors
}

\author{
Connor Donegan ${ }^{\mathrm{a}, \mathrm{b}, 1}$, Yongwan Chun ${ }^{\mathrm{a}}$, Amy E. Hughes ${ }^{\mathrm{b}}$ \\ ${ }^{a}$ Geospatial Information Sciences, University of Texas at Dallas \\ ${ }^{b}$ Population and Data Sciences, University of Texas Southwestern Medical Center
}

\begin{abstract}
This paper proposes a Bayesian method for spatial regression using eigenvector spatial filtering (ESF) and Piironen and Vehtari's (2017) regularized horseshoe (RHS) prior. ESF models are most often estimated using variable selection procedures such as stepwise selection, but in the absence of a Bayesian model averaging procedure variable selection methods cannot properly account for parameter uncertainty. Hierarchical shrinkage priors such as the RHS address the foregoing concern in a computationally efficient manner by encoding prior information about spatial filters into an adaptive prior distribution which shrinks posterior estimates towards zero in the absence of a strong signal while only minimally regularizing coefficients of important eigenvectors. This paper presents results from a large simulation study which compares the performance of the proposed Bayesian model (RHS-ESF) to alternative spatial models under a variety of spatial autocorrelation scenarios. The RHS-ESF model performance matched that of the SAR model from which the data was generated. The study highlights that reliable uncertainty estimates require greater attention to spatial autocorrelation in covariates than is typically given. A demonstration analysis of 2016 U.S. Presidential election results further evidences robustness of results to hyper-prior specifications as well as the advantages of estimating spatial
\end{abstract}

\footnotetext{
Email address: Connor.Donegan@UTDallas.edu (Connor Donegan)

${ }^{1}$ This research was supported in part by the computational resources provided by the BioHPC supercomputing facility located in the Lyda Hill Department of Bioinformatics, UT Southwestern Medical Center, TX. URL: https://portal.biohpc.swmed.edu. Connor Donegan received funding for this research from the Pioneer GIS Fellowship at UT Dallas. The authors are also grateful for feedback received from Daniel A. Griffith.
} 
models using the Stan probabilistic programming language.

Keywords: Spatial regression, spatial filtering, Bayesian inference, Stan.

\section{Introduction}

The development of spatial regression models is driven by two primary concerns. The first is that spatially referenced data, like time series data, violate the assumption of exchangeability of observations embedded in classical regression techniques as indicated by the presence of spatial autocorrelation (SA) in model residuals. Correlated observations provide less information than would the same number of uncorrelated observations, resulting in higher observed variance and greater uncertainty of parameter estimates (Griffith, 2011). A second motivation, particularly in ecology, geostatistics generally and public health research, is that spatial patterns in outcome variables may themselves be of primary interest. By incorporating evidence of spatial structure in the outcome variable into the model, spatial regression can identify areas of unexplained and elevated (or depressed) probability of event occurrence which may inform subsequent investigations.

Eigenvector spatial filtering (ESF) models incorporate spatial structure into the linear predictor of any generalized linear model (GLM) with a linear combination of the eigenvectors from a spatial connectivity matrix. Roughly concurrent with similar advances in philogenetic and ecological modeling (Diniz-Filho et al., 1998; Borcard and Legendre, 2002), Griffith (2000) introduced ESF in part as a means to circumvent the difficulties pertaining to the estimation of non-normal probability models with positive SA. ESF methodology has since been extended to address problems of network autocorrelation (Chun, 2008), multi-level models (Park and Kim, 2014; Hu et al., 2018), spatially-varying coefficients (Murakami et al., 2017), spatio-temporal autocorrelation (Griffith et al., 2019) and a variety of problems in ecological modeling (see Griffith et al., 2019, xiii-xiv).

However, the estimation of spatial filters still poses significant challenges. Spatial filters are most often estimated by means of stepwise selection procedures (Tiefelsdorf and Griffith, 2007; Chun et al., 2016). While the vicissitudes of stepwise selection are attenuated by the fact that all candidate eigenvectors are mutually orthogonal (Griffith, 2012), absent a model averaging procedure variable selection methods are unable to account for parameter uncertainty deriving from uncertainty in model selection (LeSage and Pace 
2009, Ch. 6; Gargallo et al. 2018). In a simulation study comparing spatially varying coefficient models, Oshan and Fotheringham (2018) raise concerns with multiple hypothesis testing and find that ESF estimated by stepwise selection overfit the data. Alternative methods for estimating spatial filters which seek to address some or all of these concerns include the lasso (Seya et al., 2015), an empirical Bayes random effects approach (Murakami and Griffith, 2015) and a Bayesian adaptive sampling algorithm (Gargallo et al., 2018).

This article draws on methods for high-dimensional inference to propose a Bayesian estimation procedure that avoids fitting multiple model specifications. Hierarchical shrinkage priors are normal distributions with scale parameters that adapt to the data such that posterior distributions of strong signals float towards their maximum likelihood estimate while weak signals are subject to aggressive shrinkage but without ever collapsing onto zero (see Polson and Scott, 2010). This article aims to demonstrate that Piironen and Vehtari's (2017) regularized horseshoe (RHS) prior is a computationally efficient and high performing prior distribution for the coefficients of the spatial filter.

This article first explains why the calculation of uncertainty remains a key challenge facing ESF and then introduces the proposed Bayesian model (RHS-ESF). Results from a large simulation study are then presented as arbiter of conceptual disputes found in the literature, particularly regarding the interpretation of variance inflation caused by eigenvectors which are correlated with covariates. The study is designed to evaluate the reliability of the RHS-ESF model relative to alternative ESF methods as well as the restricted spatial regression (RSR) framework (Reich et al., 2006; Hughes and Haran, 2013) and the simultaneous autoregressive (SAR) error model under a variety of SA conditions. Finally an analysis of 2016 Presidential election data demonstrates implementation of the RHS-ESF model while drawing on novel insights derived from the simulation study.

\section{ESF regression: current methods and limitations}

\subsection{ESF theory}

ESF regression incorporates spatial autocorrelation into the linear predictor of a GLM with a set of spatially-structured, synthetic control variables from a spectral decomposition of the spatial connectivity matrix. If $y_{i}$ is a normally distributed set of spatially autocorrelated observations taken at 
locations $i=1,2, \ldots, n$, then following Griffith (2011, Theorem 1-a) we may view $y_{i}$ as a mixture of normal distributions $y_{i}=y_{i}^{*}+\theta_{i}$ where $y_{i}^{*} \sim \mathrm{N}\left(\mu_{i}, \sigma^{2}\right)$ is a spatially unstructured (exchangeable) process and $\theta_{i} \sim \mathrm{N}\left(0, \omega^{2}\right)$ is a spatially structured (ordered) process. Then

$$
\boldsymbol{y} \mid \boldsymbol{\mu}, \nu \sim \mathrm{N}\left(\boldsymbol{\mu}, \nu^{2} \boldsymbol{I}\right), \nu^{2}=\sigma^{2}+\omega^{2}
$$

This conceptualization highlights how SA inflates observed variance and, while the expectation of the mean is unaffected by SA, failure to model spatial structure in any finite sample tends to produce larger estimation errors. $^{2}$ To estimate $\theta_{i}$ and recover $y_{i}^{*}$, ESF regression takes the eigenfunctions of a transformed spatial connectivity matrix such as $\boldsymbol{C}$, where $c_{i j}=1$ if polygons $i$ and $j$ are neighbors and all $c_{i i}=0$. With projection matrix $\boldsymbol{M}=\left(\boldsymbol{I}-\mathbf{1 1}^{\prime} / n\right)$, where $\boldsymbol{I}$ is the identity matrix and $\mathbf{1}$ an n-by-one vector of ones, the eigenfunction decomposition of the matrix $\boldsymbol{M C M}$ (which appears in the numerator of the Moran coefficient (MC) (Cliff and Ord, 1981, 42) produces $n$ mutually orthogonal, zero mean eigenvectors $\boldsymbol{E}$ and associated eigenvalues $\boldsymbol{\Lambda}$. Each $\boldsymbol{E}_{i}$ represents a distinct pattern of potential SA, with degree of SA indexed by its eigenvalue $\lambda_{i}$. Figure 1 maps a selection of these eigenvectors based on a connectivity matrix representing the 100 counties of North Carolina.

Griffith and Chun $(2014,1482)$ summarize the interpretation of $\boldsymbol{E}$ :

The first eigenvector, $\boldsymbol{E}_{1}$, is the set of real numbers that has the largest $\mathrm{MC}$ value achievable by any set of real numbers for the spatial arrangement defined by the spatial weight matrix $\boldsymbol{C}$; the second eigenvector, $\boldsymbol{E}_{2}$, is the set of real numbers that has the largest achievable $\mathrm{MC}$ value by any set that is uncorrelated with $\boldsymbol{E}_{1}$; the third eigenvector, $\boldsymbol{E}_{3}$, is the set of real numbers that has the largest achievable MC value by any set that is uncorrelated with both $\boldsymbol{E}_{1}$ and $\boldsymbol{E}_{2}$ and so on through $\boldsymbol{E}_{n}$, each eigenvector achieving the largest $\mathrm{MC}$ that remains uncorrelated with the preceding $n-1$ eigenvectors.

As illustrated in Figure 1, the first eigenvector represents a strong, largescale pattern of SA while eigenvectors corresponding to lower levels of SA

\footnotetext{
${ }^{2}$ From sampling theory, the standard error of the mean increases from $\frac{\sigma}{\sqrt{n}}$ for $\boldsymbol{y}^{*}$ to $\frac{\sigma+\omega}{\sqrt{n}}$ for $\boldsymbol{y}$.
} 

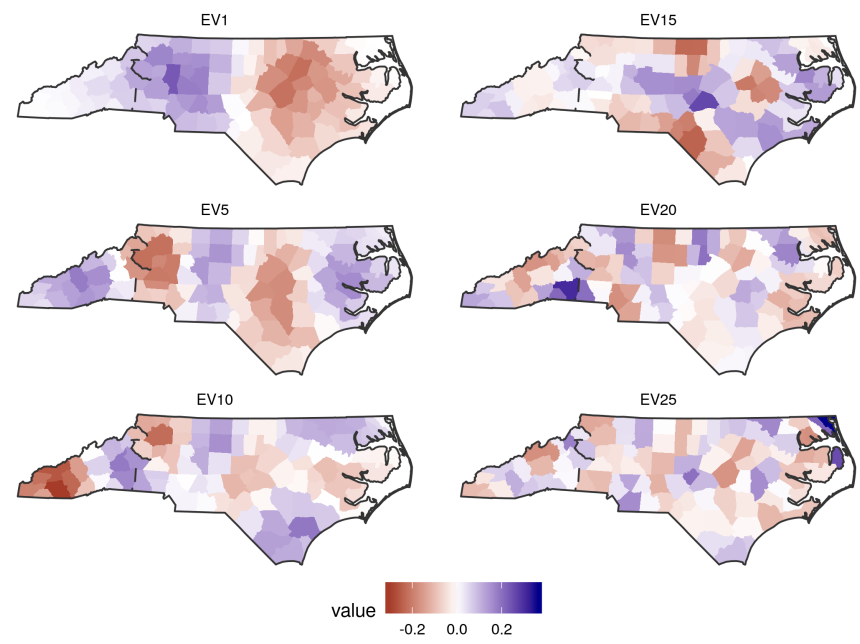

Figure 1: A selection of eigenvectors from the transformed spatial connectivity matrix of 100 counties of North Carolina.

depict smaller-scale map patterns. If the spatially structured component $\boldsymbol{\theta}$ can be estimated through a linear combination of these eigenvectors $\boldsymbol{E} \boldsymbol{\beta}_{\boldsymbol{E}}$, where $\boldsymbol{\beta}_{\boldsymbol{E}}$ is a vector of $n$ unknown coefficients, then the data can be modeled as

$$
\boldsymbol{Y} \mid \boldsymbol{\mu}, \boldsymbol{\beta}_{\boldsymbol{E}}, \sigma^{2} \sim \mathrm{N}\left(\boldsymbol{\mu}+\boldsymbol{E} \boldsymbol{\beta}_{\boldsymbol{E}}, \sigma^{2} \boldsymbol{I}\right)
$$

\subsection{Current methods for estimating spatial filters}

In practice, strong prior information on SA and spatial filters is used to reduce the dimensionality of $\boldsymbol{\beta}_{\boldsymbol{E}}$. Since most applications are concerned with positive SA, the eigenvectors representing negative SA (whose eigenvalues are negative) are not considered and their coefficients are set to zero. The problem can be simplified further by the exclusion of eigenvectors that represent trace amounts of $\mathrm{SA}$, such as by dropping all eigenvectors for which $\lambda_{i} / \lambda_{\max }<T$, with threshold $T$ set at or below 0.25 . For the counties of North Carolina, for example, this practice leaves twenty-four eigenvectors with coefficients to estimate. The most common estimation procedure takes the remaining eigenvectors to be a candidate set and applies a stepwise variable 
selection procedure to identify a final subset of eigenvectors to include and accepts the maximum likelihood estimate of their coefficients. The stepwise selection procedures may take information criteria (AIC or BIC), coefficient $p$-values, residual sum of squares or residual SA as their objective function (Chun et al., 2016; Tiefelsdorf and Griffith, 2007). Alternatively, Pace et al. (2013) argue that a fixed number of eigenvectors may be included based only on tessellation size. Outside of the variable selection paradigm, Murakami and Griffith (2015) apply residual maximum likelihood (REML) to estimate the candidate eigenvector coefficients as a set of random effects (RE-ESF).

\subsection{Limitations of current methods}

Concerns with automatic variable selection procedures are well known (Chatfield, 1995) and generally apply here. Of particular concern is that all candidate eigenvectors coefficients have non-zero prior probability for inclusion in the model but all that fail to meet the threshold for inclusion are given coefficients of $\hat{\beta}_{E i}=\operatorname{var}\left(\hat{\beta}_{E i}\right)=0$ which self-evidently eliminates some amount of uncertainty that remains after seeing the data. A proper accounting for uncertainty in the spatial process is important not just in its own right, for the purposes of prediction and possibly cluster detection, but also is necessary for an accurate estimation of the marginal effects of covariates of interest. This section argues that a primary challenge for ESF estimation is the proper calculation of uncertainty across the high-dimensional parameter space of $\boldsymbol{C}$ 's spectral decomposition.

Recall that within the frequentist paradigm the variance of a partial regression coefficient estimate $\hat{\beta}_{j}$

$$
\operatorname{var}\left(\hat{\beta}_{j}\right)=\frac{\sigma^{2}}{\sum_{i=1}^{n}\left(\boldsymbol{X}_{j_{i}}-\overline{\boldsymbol{X}}_{j}\right)^{2}}\left(\frac{1}{1-R_{j}^{2}}\right)
$$

where $\sigma^{2}$ is the residual variance and $R_{j}^{2}$ is the $R^{2}$ statistic from a regression of $\boldsymbol{X}_{j}$ on the remaining covariates in the design matrix $\boldsymbol{X}$ (Gujarati et al., 2012, 347). The precision of $\hat{\beta}_{j}$ increases with $\sum_{i=1}^{n}\left(\boldsymbol{X}_{j_{i}}-\overline{\boldsymbol{X}}_{j}\right)^{2}$ and decreases with $\sigma^{2}$ and $R_{j}^{2}$. If $\boldsymbol{\mu}=\boldsymbol{X} \boldsymbol{\beta}_{\boldsymbol{x}}$ with $\boldsymbol{\beta}_{\boldsymbol{x}}$ a vector of unknown coefficients then any non-zero pairwise correlation between covariates and eigenvectors necessarily induces additional uncertainty into the estimate of $\boldsymbol{\beta}_{\boldsymbol{x}}$. At the same time as variable selection procedures drop some eigenvec-

tors with non-zero correlations from the model, the (unpenalized) addition of those eigenvectors most correlated with the outcome variable decreases the 
residual variance of the regression. Automatic variable selection procedures thus work systematically to minimize the standard error of regression coefficients but without accounting for all sources of uncertainty. The challenge is obviously that a high-variance model with a predetermined number of up to $n$ estimated eigenvector coefficients likewise fails to meaningfully represent the researchers state of knowledge since it ignores a great deal of prior information regarding the degree of complexity in most spatial processes and may over or under-correct for SA (Chun et al., 2016).

Such correlations between eigenvectors of a spatial connectivity matrix and covariates has been coined 'spatial confounding' (Reich et al., 2006; Hodges and Reich, 2010; Hughes and Haran, 2013). In contrast to the perspective just outlined, Hodges and Reich (2010) argue that the variance inflation caused by correlations between spatial random effects and covariates does not reflect any legitimate inferential uncertainty and can also "mess up" the fixed effects estimates obtained from a non-spatial linear model. This view motivated the search for a model in which "sample size can be discounted without distorting the fixed effect estimate" (Hodges and Reich, 2010, 331). To this end Hodges and Reich propose restricted spatial regression (RSR), a spatial filtering method which introduces the eigenvectors of $\boldsymbol{M C M}$ where $\boldsymbol{M}=\boldsymbol{I}-\boldsymbol{X}\left(\boldsymbol{X}^{\prime} \boldsymbol{X}\right)^{-1} \boldsymbol{X}^{\prime}$, so that the eigenvectors are restricted to the space orthogonal to $\boldsymbol{X}$ (Hughes and Haran, 2013). ${ }^{3}$ RSR is designed such that no correlated eigenvectors are allowed to 'steal' from the explanatory power that an OLS regression would apportion exclusively to the covariates.

To the extent that correlations between covariates and eigenvectors produce a challenge for probable inference - particularly that of simultaneously estimating a nonstationary mean and the effects of covariates with limited information - surely it should be addressed as such. One reason to remain skeptical of RSR is its substitution of a deterministic separation procedure designed to purge a source of parameter uncertainty from the model, for established inferential methods. The notion that the spatial trend component of a model should have no impact on non-spatial parameter estimates overlooks the geographic analog to Yule's (1926) "nonsense correlations" which routinely appear in nonstationary time-series data and motivates much foun-

\footnotetext{
${ }^{3}$ Tiefelsdorf and Griffith (2007) previously introduced this method but for different purposes, noting an algebraic relationship to the spatial lag model specification.
} 
dational work in spatial statistics (see "Student" [W.S. Gausset], 1914; Haining, 1991; Griffith et al., 2019, Ch. 9). This suggests that the a priori restriction of the space spanned by the spatial filter is counterproductive in the sense that it ignores useful, potentially critical information. These issues are discussed further in Sections 4.2 and 5.

Lastly, Murakami and Griffith's RE-ESF model, which was an extension of Hughes and Haran's Bayesian estimation procedure, is a highly promising development for ESF since it produces (fast) penalized estimates of $\boldsymbol{\beta}_{\boldsymbol{E}}$ with empirical Bayes techniques rather than resorting to variable selection methods. However, calculating the degrees of freedom for REML models remains a challenge. Without a proper solution $\operatorname{var}\left(\hat{\beta}_{j}\right)$ is unreliable. ${ }^{4}$

\section{High-dimensional Bayesian inference}

\subsection{Hierarchical shrinkage priors}

Sparsity-imposing priors are designed for high-dimensional regression and classification problems in which one expects only a small proportion of many available covariates to be highly relevant to the outcome variable. Hierarchical shrinkage priors are a class of sparsity priors which model a set of coefficients as a mixture of normal distributions. These "global-local scale mixtures of normals" (Polson and Scott, 2010) estimate a separate scale parameter for each coefficient $\beta_{j}$ as a product of a global scale parameter $\tau$ and a local scale parameter $\lambda_{j}$ :

$$
\beta_{j} \mid \tau^{2}, \lambda_{j}^{2} \sim \mathrm{N}\left(0, \tau^{2} \lambda_{j}^{2}\right) .
$$

A small value for $\tau$ expresses our prior expectation that most of the coefficients are near zero while $\lambda_{j}$ learns from the data and enables any given parameter to become large. As Polson and Scott (2010) summarize, "To squelch noise and shrink all of the means toward zero, $\tau^{2}$ should be small. Yet in order for large signals to override this effect, $\lambda_{j}^{2}$ must be allowed to be quite large" (5). Carvalho et al.'s (2009) horseshoe prior accomplishes this by placing a positive-valued half-Cauchy prior over $\lambda_{j}$

$$
\lambda_{j} \sim C^{+}(0,1)
$$

\footnotetext{
${ }^{4}$ The spmoran software implementation of RE-ESF (Murakami, 2019) uses equation 15 from Müller et al. (2013) to calculate the effective degrees of freedom (Daisuke Murakami, personal communication).
} 
centered on zero with scale parameter of 1 . This places most prior probability density very near zero for aggressive shrinkage of weak signals but the fat tail of the distribution enables large values to arise without being subjected to any shrinkage. The horseshoe achieves dimensionality reduction without ever causing the posterior distribution of $\hat{\beta}_{j}$ to collapse completely on zero, following the rules of probability theory.

\subsection{The regularized horseshoe prior}

Piironen and Vehtari (2017) modify the horseshoe prior to address notable limitations. Their regularized horseshoe (RHS) enables researchers to encode prior information regarding the degree of sparsity into hyperprior distributions for $\tau$ while also enabling the specification of weakly informative or regularizing priors over any large $\hat{\beta}_{j}$ (i.e. any which escape extreme shrinkage). Whereas Carvalho et al. (2009) gave $\tau$ a half-Cauchy prior $\tau \sim C^{+}(0,1)$ and Carvalho et al. (2010) allowed $\tau$ to scale with the data, $\tau \mid \sigma \sim C^{+}(0, \sigma)$, Piironen and Vehtari (2017) incorporate the scale of the data, the number of observations $n$ and the researchers expectation regarding the number of parameters far from zero, $p_{0}$, into the hyper-prior distribution

$$
\begin{gathered}
\tau \mid \sigma \sim C^{+}\left(0, s_{\tau}\right) \\
s_{\tau}=\tau_{0} \sigma=\frac{p_{0}}{D-p_{0}} \frac{\sigma}{\sqrt{n}}
\end{gathered}
$$

where $D$ is the number of parameters in the design matrix (for ESF this is the number of candidate eigenvectors). This is particularly convenient for ESF regression since we have strong prior information on the number of important eigenvectors (Chun et al., 2016). However, this helpful formula for $s_{\tau}$ only applies for normal probability models. For other models such as Poisson or binomial likelihoods $s_{\tau}$ may need to be set directly using model comparison techniques (see Section 5) and trial and error (with $s_{\tau}=1$ as in the original horseshoe a sensible starting point).

Since the original horseshoe prior "resembles a spike-and-slab with an infinitely wide slab", results are not reliable when $\lambda_{j}$ is poorly identified by the data (Piironen and Vehtari, 2017). The RHS prior addresses this by regularizing the large $\hat{\beta}_{j}$ with weakly informative Student-t "slabs." Since placing an inverse Gamma prior on the scale parameter of a normal prior distribution results in marginal Student-t priors, $\lambda_{j}$ is augmented by an auxiliary 
parameter $c$ with an inverse Gamma prior:

$$
\begin{gathered}
\beta_{j} \mid \lambda_{j}, \tau, c \sim N\left(0, \tau^{2} \tilde{\lambda}_{j}^{2}\right), \quad \tilde{\lambda}_{j}^{2}=\frac{c^{2} \lambda_{j}^{2}}{c^{2}+\tau^{2} \lambda_{j}^{2}} \\
\lambda_{j} \sim C^{+}(0,1) \\
c^{2} \mid \nu_{c}, s_{c} \sim I G(\alpha, \beta), \quad \alpha=\nu_{c} / 2, \quad \beta=\nu_{c} s_{c}^{2} / 2 .
\end{gathered}
$$

When the estimate of $\lambda_{j}$ is small, the prior scale parameter for $\beta_{j}\left(\tau^{2} \tilde{\lambda}_{j}^{2}\right)$ is near zero and $c$ remains superfluous. When $\lambda_{j}$ is large enough to overwhelm $\tau$, then the auxiliary parameter acts through $\tilde{\lambda}_{j}$ as a Student-t prior on $\beta_{j}$ with degrees of freedom $\nu_{c}$ and scale $s_{c}^{2}$.

\subsection{Bayesian ESF}

To estimate a Bayesian ESF regression (RHS-ESF) one can place an RHS prior on the eigenvector coefficients in equation 2 :

$$
\boldsymbol{\beta}_{\boldsymbol{E}} \mid \tau_{0}, \nu_{c}, s_{c} \sim \operatorname{RHS}\left(\tau_{0}, \nu_{c}, s_{c}\right)
$$

This requires user specification of three hyper-parameters and is aided by the strong prior information from existing literature on spatial filtering. The scale $s_{c}$ of the Student-t slab in equation 7 can be set nearly automatically based on the scale of the data, such as one-half the standard deviation of the outcome variable divided by the standard deviation of the eigenvectors, which conveniently all have the same scale. Any value for degrees of freedom $\nu_{c}$ ranging from, say, 10 to 20 is reasonable. Setting $\tau_{0}$ can be guided by Chun et al.'s (2016) equation for the expected number of non-zero eigenvector coefficients

$$
\begin{gathered}
p_{0}=\frac{n_{\text {pos }}}{1+\gamma} \\
\gamma=\exp \left[\frac{2.1480-\left(6.1808\left(z_{M C}+0.6\right)^{0.1742}\right)}{n_{\text {pos }}^{0.1298}+3.3534 /\left(z_{M C}+0.6\right)^{0.1742}}\right]
\end{gathered}
$$

where $n_{\text {pos }}$ is the total number of positive-valued eigenvalues and $z_{M C}$ is the residual $\mathrm{MC}$ z-score obtained from a non-spatial model. This quantity can be entered into equation 6 if the likelihood model for the data is Gaussian or Student's t. Various specifications may be compared using residual SA and Widely Applicable Information Criteria (Watanabe, 2010) (see Section 5).

The model can be estimated (and extended arbitrarily as needed) using the Stan probabilistic programming language (Carpenter et al., 2017; Stan 
Development Team, 2019) making use of Stan code provided by Piironen and Vehtari for the RHS prior. Stan is an open-source platform for specifying probability models. Samples from target posterior distributions are obtained using Hamiltonian Monte Carlo and the No-U-Turn Sampler (NUTS) (Hoffman and Gelman, 2014).

\section{Simulation study}

\subsection{Study aims and design}

The purpose of the simulation study is to evaluate the reliability of the RHS-ESF model with a Gaussian likelihood for the purposes of estimating the marginal effects of covariates and to compare results to commonly used alternative specifications. Spatially autocorrelated data was simulated from a multivariate normal distribution with a simultaneous autoregressive (SAR) process in the covariance matrix:

$$
\begin{gathered}
\boldsymbol{y} \sim \mathrm{N}_{k}\left(\boldsymbol{\mu}, \boldsymbol{Q}^{-1}\right) \\
\boldsymbol{Q}=(\boldsymbol{I}-\rho \boldsymbol{W})(\boldsymbol{I}-\rho \boldsymbol{W})^{\prime} \sigma^{2}
\end{gathered}
$$

where $\boldsymbol{\mu}$ is the length- $k$ vector, $\boldsymbol{Q}$ is a $k$-by- $k$ dimensional matrix, $\boldsymbol{W}$ is a $k$-by- $k$ row-standardized spatial connectivity matrix, $\boldsymbol{I}$ is a $k$-by- $k$ identity matrix, $\rho$ is a scalar providing the degree of spatial autocorrelation and $\sigma$ is the standard deviation of $\boldsymbol{y}$. All specifications utilized in this study have $\sigma=1$. The 100 counties of North Carolina and the queen contiguity condition were used to produce the spatial connectivity matrix. Each draw from equation 10 consists of a vector of 100 spatially correlated values.

The mean $\boldsymbol{\mu}$ of $\boldsymbol{y}$ is a linear function of two covariates:

$$
\boldsymbol{\mu}=\mathbf{1}+\beta_{1} \boldsymbol{x}_{\mathbf{1}}+\beta_{2} \boldsymbol{x}_{\mathbf{2}}, \quad \beta_{1}=0.5, \quad \beta_{2}=-0.5 .
$$

The two covariates consist of independent draws from separate, zero-mean SAR processes. Thus $\boldsymbol{y}$ contains SA which can be explained by covariates and, independent from its mean $\boldsymbol{\mu}$, additional spatially correlated variation. The degree of SA in the outcome variable $\left(\rho_{y}\right)$ and covariates $\left(\rho_{x}\right)$, respectively, were systematically varied to capture a range of SA conditions. For

each of the 25 combinations of $\rho_{y}, \rho_{x} \in\{0.1,0.3 ., 0.5,0.7,0.9\} 1,000$ sets of data were drawn for a total of 25,000 data sets. Other parameters are held constant in order to isolate the impact of $\mathrm{SA}$ on inferences. 
The following models were fit to each data set: ordinary-least squares regression (OLS), ESF regression fit by stepwise selection (STEP-ESF) with pvalue of 1 as the objective criteria, Murakami and Griffith's RE-ESF method using the spmoran R package (Murakami, 2019), the RHS-ESF model estimated using Stan and a SAR error model estimated using the spatialreg $\mathrm{R}$ package (Bivand et al., 2008). In addition, the STEP-ESF and RE-ESF models were each fit a second time using eigenvectors orthogonal to $\boldsymbol{X}$ (with the eigenvectors being specific to each data set) following Hughes and Haran's RSR theory. Finally, each of the preceding models was fit a second time with the mean spatial lag of each covariate (SLX) added to the linear predictor. The SLX term was calculated by $W X$ with $W$ a row-standardized spatial weights matrix.

Prior distributions for the Bayesian ESF models were set to be weakly informative (i.e. they are centered on zero and are flat across the region of potential parameter values given the scale of the data but taper off outside of that region) (Gelman et al., 2014, 55-56). The RHS prior were given the following hyper-priors: $\tau_{0}=.4, \nu_{c}=15, s_{c}=4$ which produces a Student-t slab centered on zero with a scale of four. In practice more than one value for $\tau_{0}$ should be tested and results generally should not be accepted if the model has not removed SA from the residuals. For this study the model residuals were tested for SA using the MC; if the MC was higher than its expected value $^{5}$ then the degree of sparsity was relaxed by setting $\tau_{0}=0.9$, the model was fit again and the results were accepted unconditionally.

Within each SA condition, the estimates of $\boldsymbol{\beta}_{\boldsymbol{x}}$ are evaluated mainly on two criteria: root-mean squared error (RMSE) $\sqrt{\frac{1}{n} \sum_{i=1}^{n=1,000}\left(\hat{\beta}_{j_{i}}-\beta_{j}\right)^{2}}$ and interval coverage defined as the proportion of $95 \%$ confidence/credible intervals that contain the true parameter value. ${ }^{6}$ Interval coverage for a properly specified model is expected to be near 950 out of each 1,000 models. A secondary criterion is efficiency, calculated as the mean width of the $95 \%$

\footnotetext{
${ }^{5}$ The formula for the expected value of the residual $\mathrm{MC}$ is $-n T R\left[\left(\boldsymbol{X}^{\prime} \boldsymbol{X}\right)^{-1} \boldsymbol{X}^{\prime} \boldsymbol{C} \boldsymbol{X}\right] /\left[(n-k)^{\prime} \boldsymbol{C} \mathbf{1}\right]$ where $T R$ denotes the matrix trace operator, the $n \times k$ design matrix $\boldsymbol{X}$ includes a column of ones and $\mathbf{1}$ is an $n \times 1$ vector of ones (Chun and Griffith, 2013, 18).

${ }^{6}$ Mean estimation error $\left(\frac{1}{n} \sum_{i=1}^{n=1,000}\left(\hat{\beta}_{j_{i}}-\beta_{j}\right)\right)$ was confirmed generally to be very small for all models $(<.01)$, confirming their unbiasedness. The highest mean errors observed were around .02 (or four percent of $\beta_{j}$ ) for the OLS and the RSR models with SLX when $\rho_{y}=.9$.
} 
intervals. For the Bayesian model the mean of the posterior distribution is used for $\hat{\beta}_{j}$ and the quantiles of the posterior distribution are used to construct the credible intervals. Finally, since $x_{1}$ and $x_{2}$ are uncorrelated with each other the reported RMSE, interval coverage and efficiency scores are the averages across $\beta_{1}$ and $\beta_{2}$.

This study design is comparable to Murakami and Griffith (2015) who draw 2,000 samples from each of 27 specified conditions. However, this study generates data from a baseline SAR model rather than the model under examination and interval coverage rates are directly evaluated. This study is far more extensive than previously published Bayesian ESF methodology papers which report drawing 100 samples from a single specification (Hughes and Haran, 2013; Gargallo et al., 2018).

\subsection{Results}

Figure 2 reports RMSE of each model specification across all SA conditions. Consider first the upper half of the figure which shows results for specifications without the SLX term. With $\rho_{y}=.1$ all models have indistinguishable RMSE, though the RMSE of STEP-ESF is slightly above the others. As $\rho_{y}$ increases the accuracy of the OLS estimates steadily deteriorate relative to all conventional spatial models. Whereas the RMSE of OLS estimates are approximately 50\% larger than the SAR model when $\rho_{y}=.7$, when $\rho_{y}=.9$ the magnitude of error is double that of the SAR model. Notably, neither of the RSR specifications manages to meaningfully improve upon OLS. The RMSE for conventional RE-ESF, RHS-ESF and SAR specifications are nearly identical for most SA conditions, though when $\rho_{y} \in\{.5, .7\}$ the SAR model registers a slight advantage. Finally, we notice that estimation error falls slightly when $\rho_{x}>0.5$. This is the result of the increased $\operatorname{var}\left(x_{j}\right)$ which SA induces; the higher variance seems to provide additional information content for the model. Yet with very strong SA in the outcome variable $\left(\rho_{y}=.9\right)$, this pattern is reversed.

The bottom panel of Figure 2 reports results for models that include the SLX term. The most notable impact is on the OLS model which produces RMSE comparable to the SAR model in all but a few SA conditions $\left(\rho_{y}=\right.$ 0.9 combined with $\left.\rho_{x}<0.7\right)$. Most surprising though is the failure of the RSR specifications to achieve comparable improvement; consequently, OLS outperforms RSR when SLX is included. Interestingly, at lower levels of $\rho_{y}$ the SLX term flattens out the RMSE trend such that high $\rho_{x}$ no longer improves the RMSE; when $\rho_{y} \in\{0.7,0.9\}$ the addition of SLX increases error 


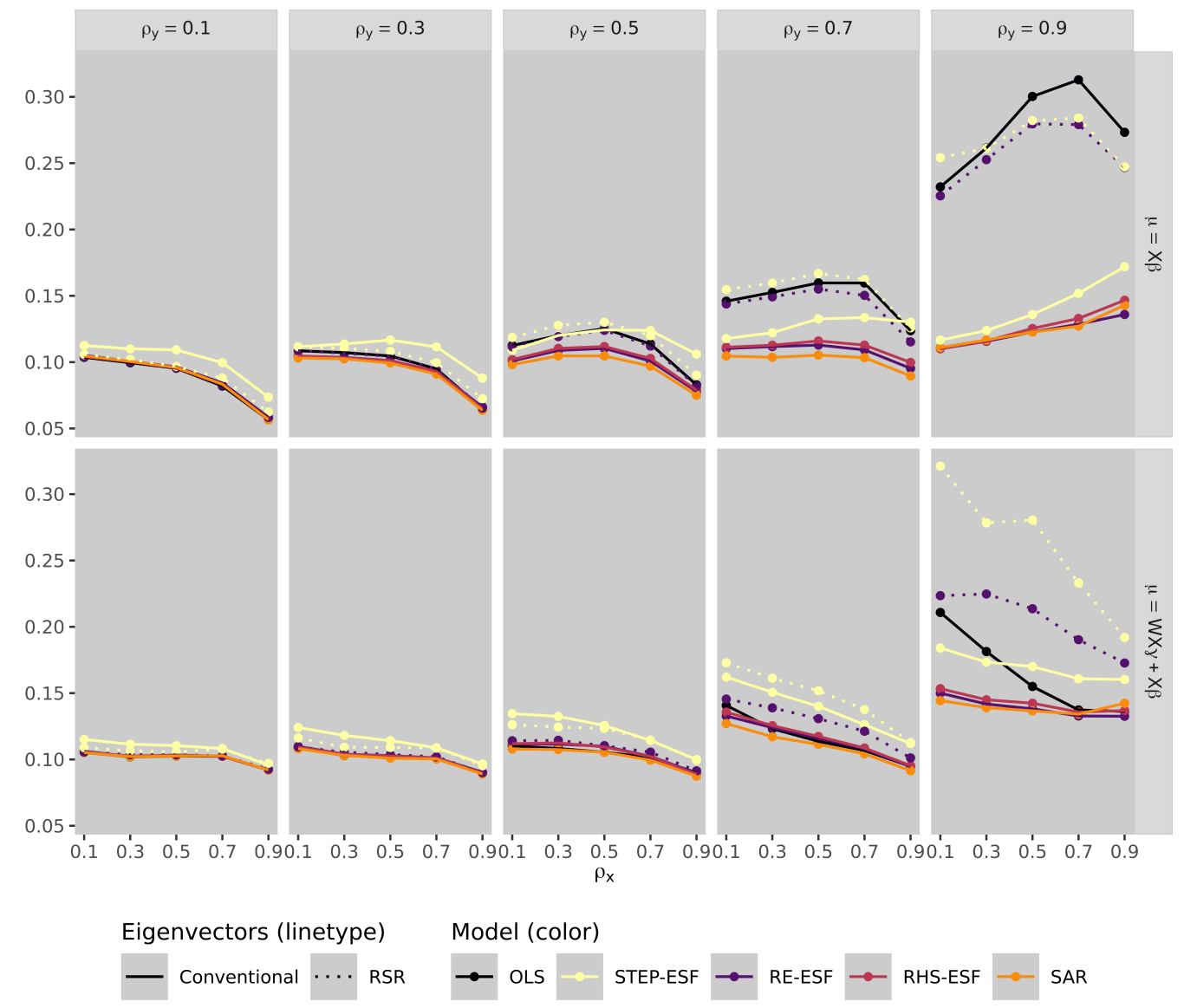

Figure 2: RMSE of $\hat{\beta}_{j}$ by model specification and SA condition.

if $\rho_{x}$ is small but does not cause harm when $\rho_{x}>0.5$. Finally, the small RMSE advantage of the SAR model over RHS-ESF and RE-ESF essentially disappears with the addition of SLX.

Whereas RMSE of the OLS model deteriorates with $\rho_{y}$, OLS interval coverage falls as $\rho_{x}$ rises and the rate at which coverage falls increases with $\rho_{y}$ (see the top panel of Figure 3 ). To greatly varying degrees, all of the spatial models exhibit this trend. STEP-ESF and RE-ESF both outperform OLS at high levels of $\rho_{y}$ but are nonetheless unable to reach $95 \%$ coverage even when $\rho_{x}=0.1$. RHS-ESF and SAR specifications share remarkably similar interval coverage: they are both on target when $\rho_{x}<0.5$ and otherwise they are near $2.5 \%$ below target. Yet when $\rho_{y}=\rho_{x}=0.9$ even SAR and RHS-ESF exhibit 
miserable coverage $(<82.5 \%)$. RSR specifications are once again inferior to OLS and often considerably so.

Turning to the bottom panel of Figure 3, the addition of the SLX term has a dramatic impact on interval coverage rates: OLS coverage now exceeds $95 \%$ and the trend of declining coverage with rising $\rho_{x}$ disappears for all ESF specifications (with the exception of $\rho_{y}=\rho_{x}=0.9$ ). RHS-ESF maintains coverage of $95 \%$ across nearly all SA conditions, SAR coverage is slightly below that of RHS-ESF for $\rho_{x}>0.5$ and the RSR specifications still generally perform more poorly than conventional ESF specifications.

Figure 4 provides some insight into the patterns in coverage rates. It reveals that as $\rho_{x}$ and thus $\operatorname{var}(x)$ increase, all of the intervals become narrower (following the logic of equation 3). Addition of the SLX term counteracts this pattern, dampening but not eliminating the trend. Further, we can see that the SAR and RHS-ESF specifications are far more efficient than OLS. Interval widths of RHS-ESF are slightly wider than those of the SAR model in most conditions, which explains the slight advantage in interval coverage rates apparent in certain SA conditions. The STEP-ESF and RE-ESF specifications, however, produce uncertainty estimates that are consistently even narrower (with and without RSR) and which we now know are generally over-optimistic.

Figure 5 provides pairwise comparisons of the spatial filters estimated by each ESF method using conventional eigenvectors in two different SA conditions: $\rho_{y}=\rho_{x}=0.3$ and $\rho_{x}=\rho_{y}=0.7$. Since neither RE-ESF nor RHS-ESF select eigenvectors from the candidate set but, instead, provide coefficient estimates for each, here we compare the estimated spatial filter $\theta_{i}$ at each observation. For the Bayesian model, the mean of the posterior distribution of $\theta_{i}$ is taken as the point estimate. The difference in estimates across methods is greatest with low SA: Figure 5a shows that for $\rho_{x}=\rho_{y}=$ 0.3 the Bayesian and empirical Bayes methods both shrink the estimates towards zero rather aggressively, such that the STEP-ESF method is actually less sparse despite fixing a number of coefficients at zero. With moderately strong SA $\left(\rho_{x}=\rho_{y}=0.7\right)$ the estimates of $\theta_{i}$ show less systematic difference across methods - the red smoothed fit lines in Figure $5 \mathrm{~b}$ are closer to the perfect fit line $y=x$ and the magnitude of variation around the fit lines is notably less. 


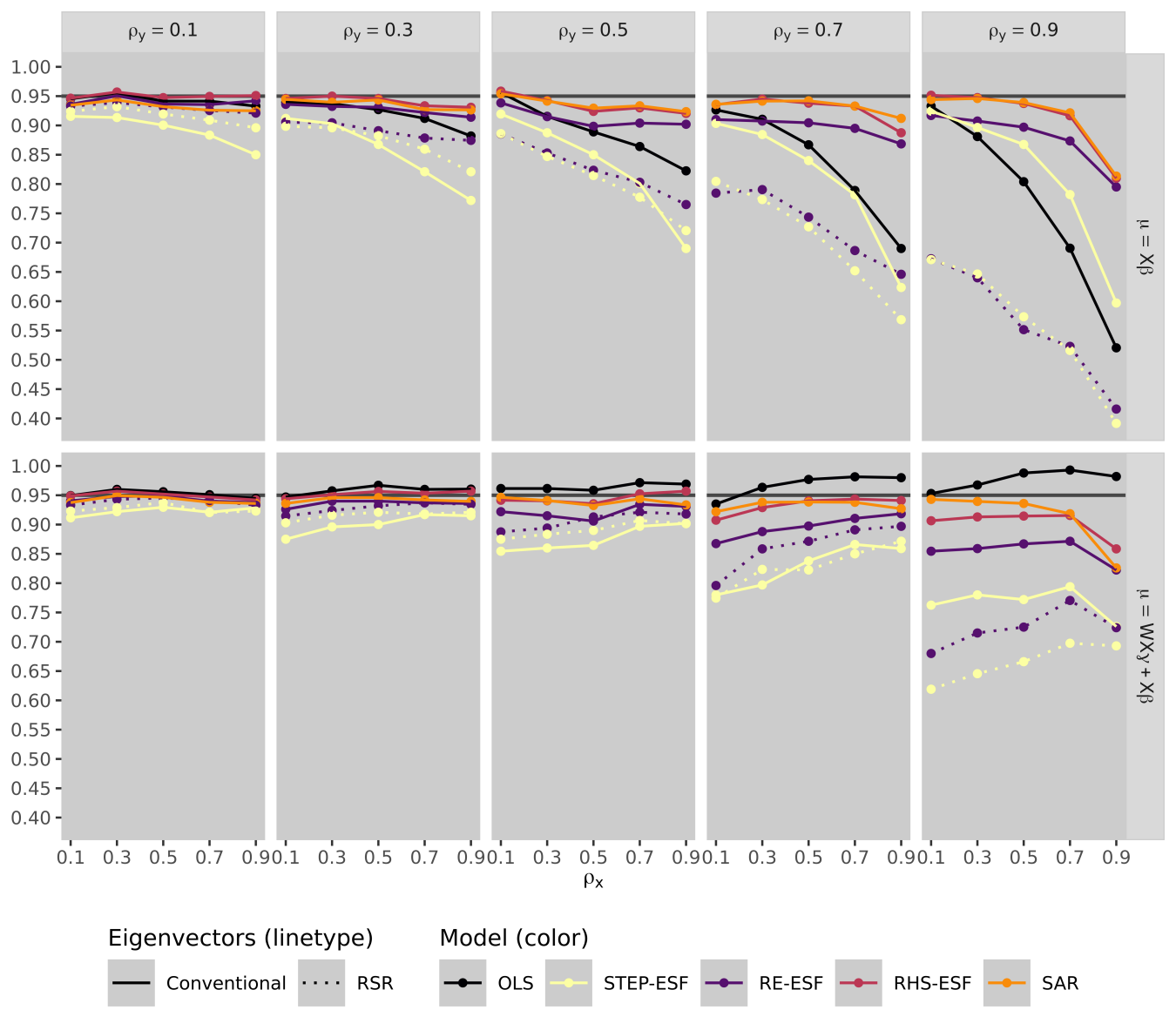

Figure 3: Proportion of $95 \%$ confidence/credible intervals for $\hat{\beta}_{j}$ containing the true parameter value by model specification and SA condition.

\subsection{Discussion}

Results from the simulation study confirm the main concerns discussed in the theoretical discussion of Section 2.3, reflect well on the proposed Bayesian ESF model and provide additional insight. As predicted, the STEP-ESF model and the RSR specifications systematically underestimated the degree of uncertainty present in parameter estimates, as did RE-ESF. While STEPESF and RE-ESF greatly improved upon OLS estimates in terms of RMSE, RSR led to high-error point estimates with overly confident standard errors, a most undesirable combination. This is not surprising given that RSR intends to maintain OLS estimates, which are known to have higher error than spatial 

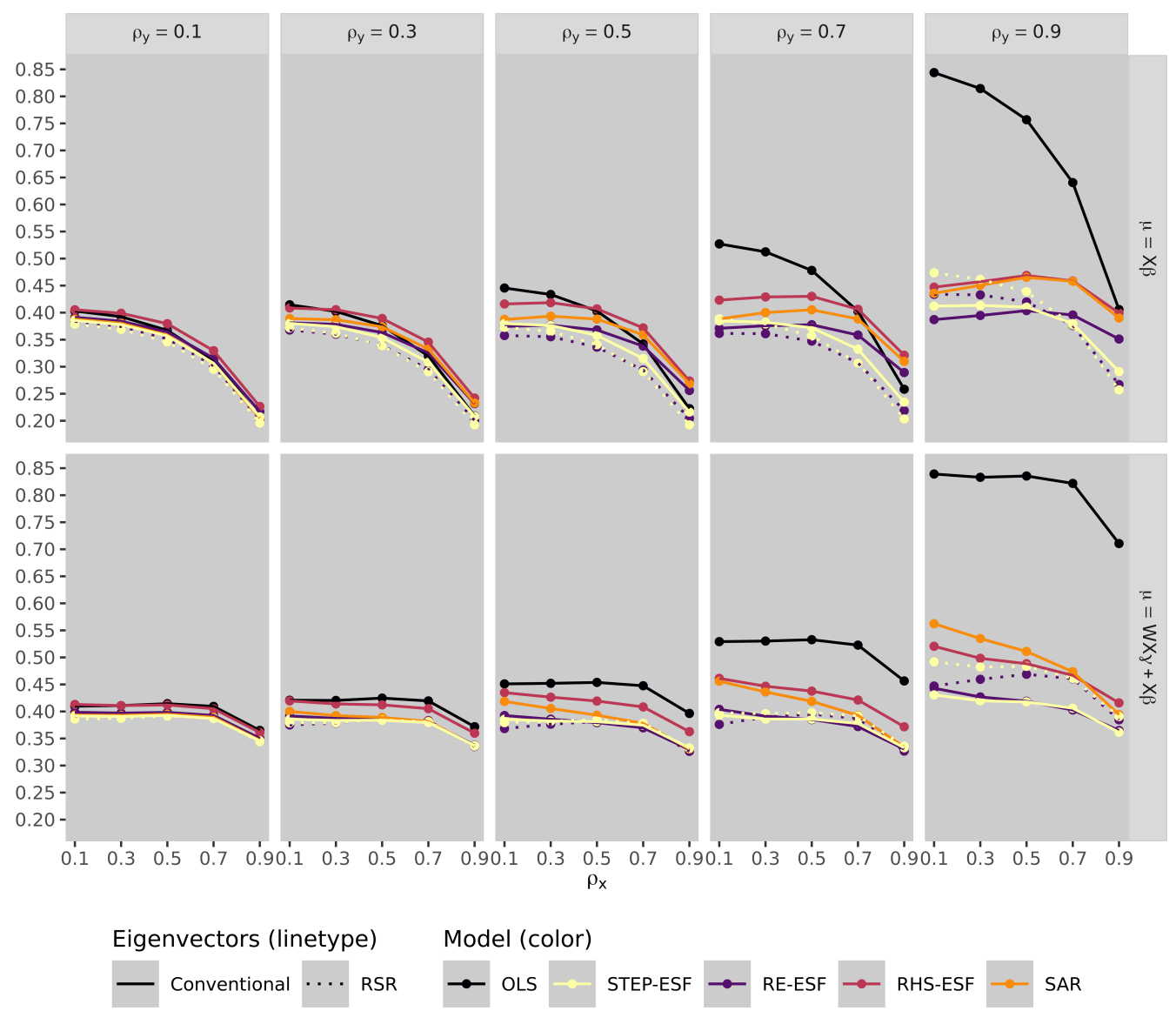

Figure 4: Mean width of $95 \%$ confidence/credible intervals for $\hat{\beta}_{j}$ by model specification and SA condition.

models when SA is present. The overly confident uncertainty estimates are simply a result of adding uncorrelated eigenvectors to the linear predictor such that $\sigma^{2}$ is decreased without impacting $R_{j}^{2}$.

On the positive side, RHS-ESF matched and sometimes exceeded the performance of the SAR model in terms of RMSE and interval coverage without a notable loss of efficiency. It is important to note that when the covariates were embedded with moderate to strong SA none of the models were able to reach the target interval coverage without the inclusion of the SLX term. Considering both RMSE and interval coverage, the results indicate that when a covariate has $\rho>0.5$ it is important to include its mean spatial lag in the 

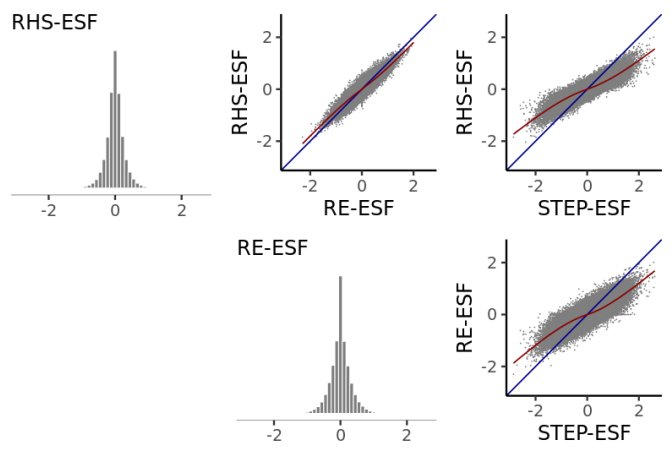

STEP-ESF

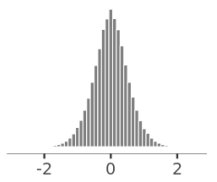

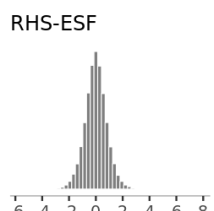

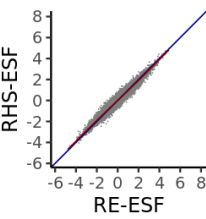

RE-ESF
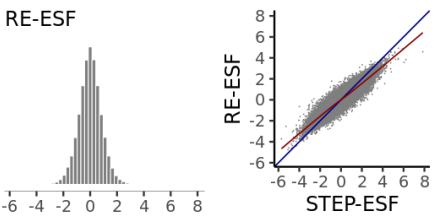

STEP-ESF

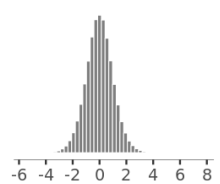
(a) $\rho_{x}=\rho_{y}=0.3$
(b) $\rho_{x}=\rho_{y}=0.7$

Figure 5: Pointwise comparisons of estimated spatial filters at each observation (i.e. county) at specified levels of spatial autocorrelation, with $y_{i}=X_{i} \beta+\theta_{i}$. As each spatial filter $\boldsymbol{\theta}$ is an $n$-length vector $(n=100)$ and each plot visualizes the spatial filters fit to 1,000 simulated data sets, each plot contains $1,000 \times n$ estimated values of $\theta_{i}$. For the Bayesian model, the mean of the posterior distribution of $\theta_{i}$ is used as the point estimate. The blue line is the theoretical perfect fit line $y=x$, the red line is a smoothed fit line $y \sim s(x)$ using a cubic basis spline (Wood, 2017). 
model in order to obtain reliable uncertainty estimates. For dependent variables with very strong SA a higher threshold should perhaps be set, e.g. $\rho>0.65$. This is true even when spatial spillover effects are not theorized to be relevant (cf. LeSage and Pace, 2009; Pace et al., 2013). Indeed, SLX appears nowhere in the data generating model used for this study. The SLX term may be interpreted as a control variable or nuisance parameter that corrects $\operatorname{var}(\hat{\beta})$ (or the posterior $\mathrm{p}(\beta \mid y, x, W)$ ) for the reduced information content of $x$ relative to an uncorrelated set of observations with the same variance. $^{7}$

Computations for RHS-ESF models were performed on a high-performance computing cluster and distributed across 16 256-gigabyte nodes. Computation time was approximately 20 minutes per 1,000 models for a total of approximately 16.6 hours for 50,000 RHS-ESF models. All of the non-Bayesian models were fit on a Dell XPS 13 laptop computer with Intel Core i7-8565U CPU @ $1.8 \mathrm{GHz}$ with computations spread across eight logical cores, completing in one hour and forty-six minutes. The same laptop requires two hours and ten minutes to estimate 1,000 RHS-ESF models. Without access to a high performance computing cluster and an MCMC sampler as efficient as Hamiltonian Monte Carlo, the cost of obtaining these results would have been prohibitive. Whereas Stan is notably inefficient for sampling from SAR and spatial lag models (Wolf et al., 2018), the RHS-ESF model is highly efficient for moderately sized data sets.

\section{Demonstration: analyzing unemployment and the Trump vote}

This section provides a brief demonstration of the RHS-ESF model through an analysis of 2016 Presidential election results in the state of Ohio. In particular, we consider the relationship between local unemployment estimates and support for Donald Trump relative to the average Republican (GOP) two-party vote share (Republican/(Republican + Democratic)) from 2004 to 2012. The analysis is relevant for evaluation of competing explanations of the international resurgence of exclusionary populism

\footnotetext{
${ }^{7}$ As Jaynes (2003) emphasizes, probability theory "is concerned with logical connections, which may or may not correspond to causal physical influences" (60). Neighboring values of $x_{i}$ may provide information about $y_{i}$ independently of any causal relations (e.g. 'spillover effects') from neighboring $x$ values to $y_{i}$.
} 
which often relate deteriorating economic conditions for low-skilled workers to perceived social-status threat within the dominant ethnic group (see Dorn et al., 2016; Monnat, 2016; Agnew and Shin, 2019). Our primary focus is on questions related to fitting Bayesian ESF models. We compare the Bayesian ESF model primarily to ESF fit by stepwise selection and highlight the flexibility afforded by our use of the Stan programming language.

Table 1 provides summary statistics of the data. Growth in the GOP vote share (gop_growth) is defined as Donald Trump's 2016 Presidential two-party vote share minus the historic GOP average (historic_gop) (MIT Election Data and Science Lab, 2018). The independent variables included are the natural logarithm of population density, percent of the population age 25 and older with a college degree, percent nonhispanic white (U.S. Census Bureau, 2016; Walker, 2019) and the local area unemployment estimate (Bureau of Labor Statistics, 2016). The variables show moderate to strong SA with the dependent variable and the main explanatory variable of interest having $\rho \geq 0.8$.

In addition to partisan leanings, these variables capture the main socioeconomic divisions in Ohio - the urban-rural divide, race, and a social fabric stratified by education (Case and Deaton, 2020). In an ordinary linear model these variables (excluding unemployment) account for $88 \%$ of the variation in GOP growth. The largest variance inflation factor (VIF) value is 5.5 (log population density). Each of the variables display, to varying degrees, elements of an underlying spatial structure that ties together Appalachian Ohio (the environmental and cultural region bordering Kentucky, West Virginia and Pennsylvania), metropolitan Cincinnati, Columbus, Toledo and Cleveland, and the remaining agricultural counties (see Figure 6). Following lessons from the simulation study, we employ a conventional spatial filter to identify variation that exceeds major SA pattern and include SLX terms for all independent variables with $\rho \geq 0.6$.

The added variable plots in Figure 7 support the use of (log-) linear relationships for all of the covariates (for the purposes of this demonstration) but also reveal heteroskedasticity and major outliers. Influential outliers include Athens County, home to Ohio University and the only heavily Democratic county in Appalachian Ohio, and Belmont County where Trump outperformed the historic average by 22 points. We use a Student's t likelihood function for robust estimates (Geweke, 1993; Gelman et al., 2014), using a Gamma prior distribution for the degrees of freedom $\nu \sim \mathrm{G}(2,0.1)$.

A potential concern with the RHS-ESF model is its reliance on hyper- 
Table 1: Summary statistics for Ohio election data including $\rho$ estimate from an intercept only SAR model.

\begin{tabular}{rcccccc}
\hline & Mean & SD & Skewness & Min & Max & $\rho$ \\
\hline gop_growth & 10.71 & 6.24 & -0.39 & -6.45 & 27.08 & 0.80 \\
historic_gop & 57.18 & 9.16 & -0.49 & 31.94 & 75.07 & 0.60 \\
$\log$ (pop.density) & 5.04 & 0.99 & 0.95 & 3.45 & 7.92 & 0.70 \\
white_nonhispanic & 90.45 & 7.51 & -2.04 & 59.98 & 97.95 & 0.32 \\
college_educated & 12.28 & 5.08 & 1.48 & 5.13 & 33.59 & 0.69 \\
unemployment & 5.50 & 1.46 & 1.01 & 3.30 & 11.10 & 0.83 \\
\hline
\end{tabular}
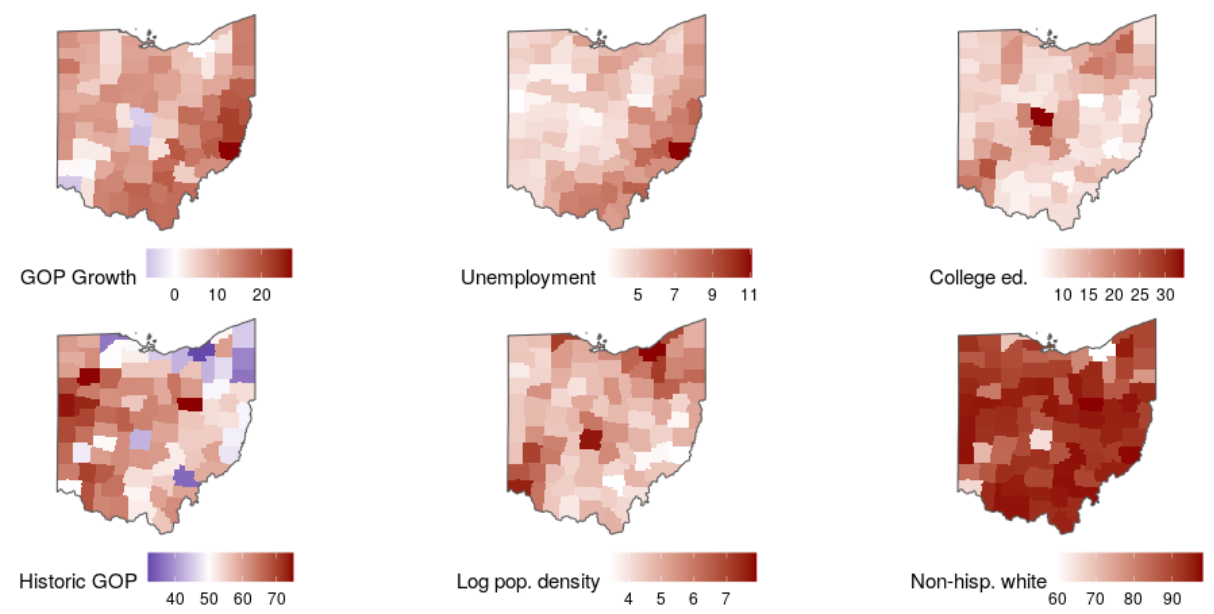

Figure 6: Maps of the dependent and independent variables.

prior specifications. If posterior distributions tend to be highly sensitive to prior specifications then reliability and ease of use suffers. Results from the simulation study already suggest this is not the case, as favorable estimates were obtained when hyper-priors were set in crude fashion. Figure 8 reinforces this conclusion through a comparison of various values of $\tau_{0}$ ranging from 0.01 to 3.01 for the Ohio election model. In practice values of $\tau_{0}$ greater than one are not often of interest since $\tau$ is a scale reduction factor. We aim for models with minimum WAIC without over-correcting for SA. As $\tau_{0}$ increases less shrinkage is imposed on the spatial filter thereby reducing residual SA. WAIC values are practically equivalent between roughly 0.3 and 1, increasing thereafter. The right-hand panel of Figure 8 reports the pos- 

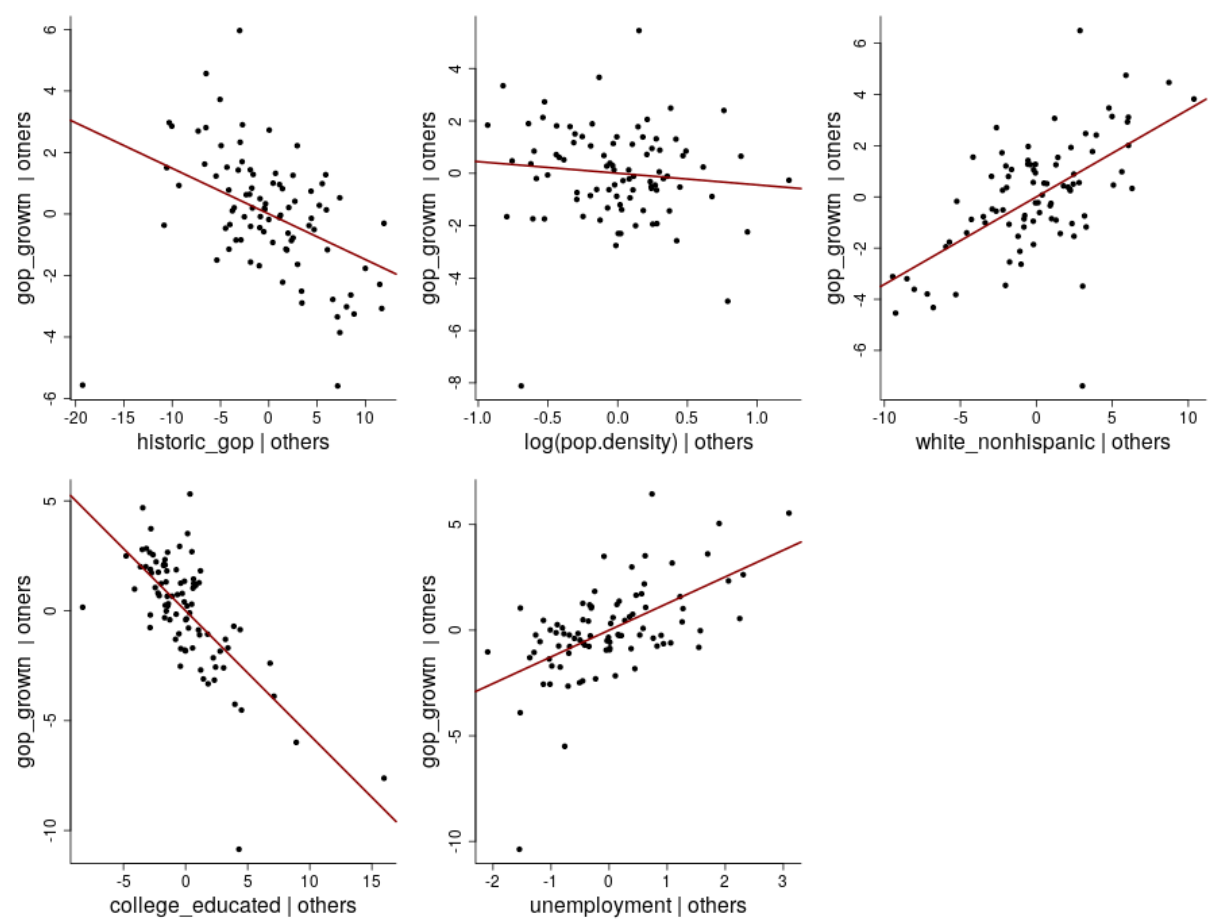

Figure 7: Added variable plots. On the x-axes are the residuals from a regression of the respective $x_{i}$ on all other independent variables, on the y-axes are the residuals from the regression of GOP growth on all of the independent variables except for $x_{i}$.

terior mean and $95 \%$ credible interval for the coefficient on unemployment $\beta_{\text {unemp }}$, which is stable within the range of acceptable values of $\tau_{0}$. With 23 candidate eigenvectors (using threshold $T \geq 0.25$ ), Equation 9 produces a value of $p_{0}=19$ as the expected number of non-zero eigenvector coefficients; plugging this into equation 6 provides a hyper-prior value of $\tau_{0}=0.51$. Our sensitivity test similarly suggests any value between 0.4 and 1 is acceptable.

All covariates were centered and scaled, so that coefficients represent expected change in GOP growth per one standard deviation change in $x$. To fit the RHS-ESF model, 5, 000 samples were drawn from each of 5 MCMC chains, discarding the first 2,500 of each as warm-up. This provided a total of 12,500 samples from the posterior distribution. Sampling and postprocessing completed in 18 seconds distributed across 5 cores on a laptop computer. Figure 9 reports test statistics to evaluate the MCMC chains. Serial correlation in the chains reduces effective sample size; from 9a we 


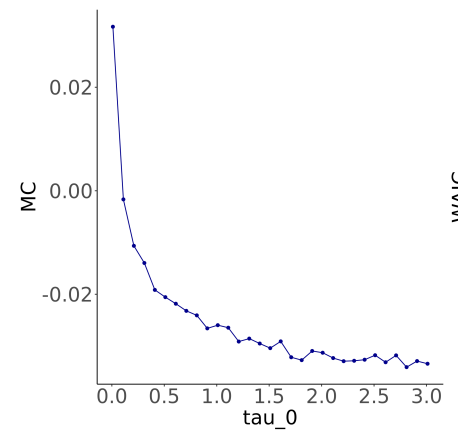

(a) Residual SA

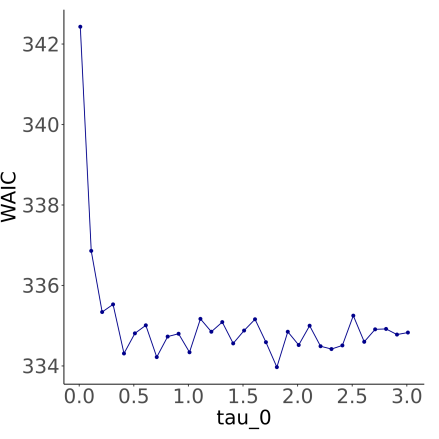

(b) WAIC

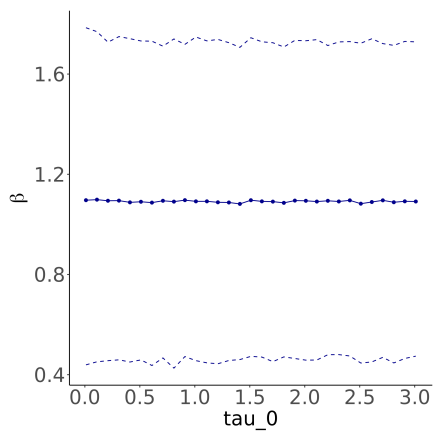

(c) Posterior $\mathrm{p}(\beta \mid$.)

Figure 8: Model sensitivity to $\tau_{0}$ hyper-prior specification: residual SA, WAIC and posterior mean and $95 \%$ quantile (credible) interval for the marginal effect of unemployment in the Ohio election model.

find the smallest effective sample size of the parameters in our model is near $0.25 * 12,500=3,125$. Figure $9 \mathrm{~b}$ reports the split $\hat{R}$ diagnostic to evaluate stationarity within chains and convergence across chains (Gelman et al., 2014, 284-285). Values are very near one indicating the chains are stationary and converged to a single distribution. In 9c, yrep are draws from the posterior predictive distribution of the model (see Gelman et al., 2014, 143-153) - that is, from a Student's t distribution with parameters $\nu$, $\boldsymbol{\mu}=\alpha \mathbf{1}+\boldsymbol{W} \boldsymbol{X} \boldsymbol{\gamma}+\boldsymbol{X} \boldsymbol{\beta}_{\boldsymbol{x}}+\boldsymbol{E} \boldsymbol{\beta}_{\boldsymbol{E}}$, and $\sigma$ drawn from their respective posterior distributions. The overlay shows that the posterior predictive distribution reproduces the primary contours of the observed data.

While estimates from the STEP-ESF model are influenced by the outlying observation (Athens County), the robust RHS-ESF model essentially down-weights the observation (see Figure 10) leading to a higher residual for Athens but robust parameter estimates. However, Figure 11 reveals how the STEP-ESF spatial filter absorbed much of the influence of Athens. A comparison of estimates across models (Tables 2 and 3) shows that the most prominent impact of the outlier is on the unemployment estimate. Whereas a SAR model provides an estimate of $\hat{\beta}_{\text {unemp }}=1.70$ which is near the OLS estimate $\hat{\beta}_{\text {unemp }}=1.67$, STEP-ESF produces an estimate of $\hat{\beta}_{\text {unemp }}=1.21$. This is much closer to the robust Bayesian model which has posterior mean $\mathrm{E}\left[\beta_{\text {unemp }}\right]=1.10$.

Whereas STEP-ESF selected 17 of the eigenvectors, the RHS-ESF model provides posterior probability distributions for each eigenvector coefficient 


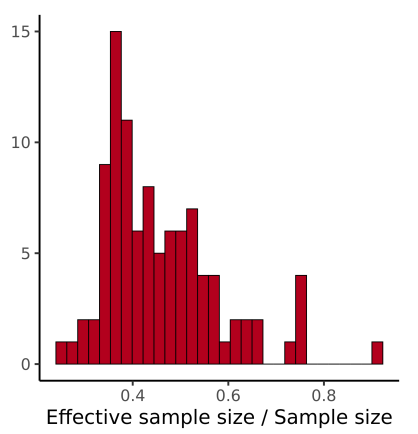

(a) Autocorrelation

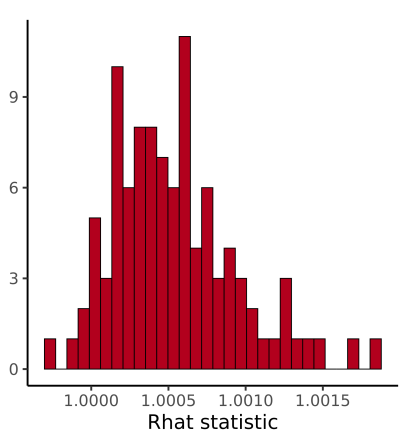

(b) Convergence

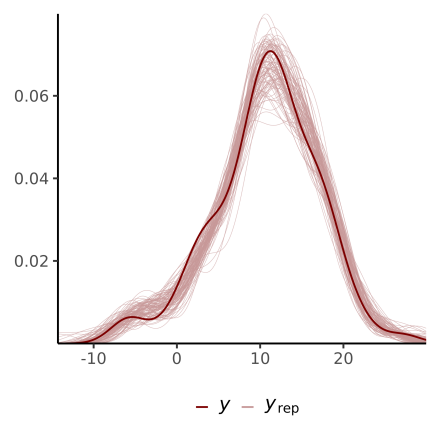

(c) Posterior predictive check

Figure 9: MCMC sampler diagnostics and posterior predictive check for the RHS-ESF Ohio election model. The histograms gather diagnostic test statistics (effective sample size and $\hat{R}$ ) calculated for model parameters (the intercept, $\beta, \sigma, \nu$ and the spatial filter $\theta)$. Figure $9 \mathrm{c}$ shows 100 draws from the posterior predictive distribution of the model. Plots were generated by the bayesplot R package (Gabry et al., 2019).

(see Figure 12). Most of the posterior modes are at zero while some place nearly all credibility on large values (the $5^{\text {th }}$ and $14^{\text {th }}$ eigenvectors). The presence of skewed and multi-modal probability densities explains why stepwise selection performed worse than RHS-ESF in the simulation study - when eigenvectors are dropped from the model, these long tails constitute unaccounted for uncertainty; when selected, maximum likelihood estimates ignore the probability density around zero which leads to noisier spatial filter estimates (as observed in Figure 5). The posterior probability distribution of $\theta_{i}$ is, conditional on all data and prior probabilities, $\mathrm{p}\left(\theta_{i} \mid.\right)=\sum_{j=1}^{k} E_{i, j} \mathrm{p}\left(\beta_{E j} \mid.\right)$ which tends to be unimodal and (nearly) symmetric (see Figure 13).

The final results provide evidence in favor of the view that poor economic prospects among peripherally located whites contributed to the collective appeal of the Trump campaign inside what was part of the Democratic "Blue Wall". Any further substantive interpretation requires additional contextual and theoretical discussion which is beyond the scope of this demonstration.

\section{Conclusion}

This study has argued that key challenge for spatial filtering-based regression is the accurate quantification of uncertainty in the spatial component of the model and, by extension, other model parameters such as the 


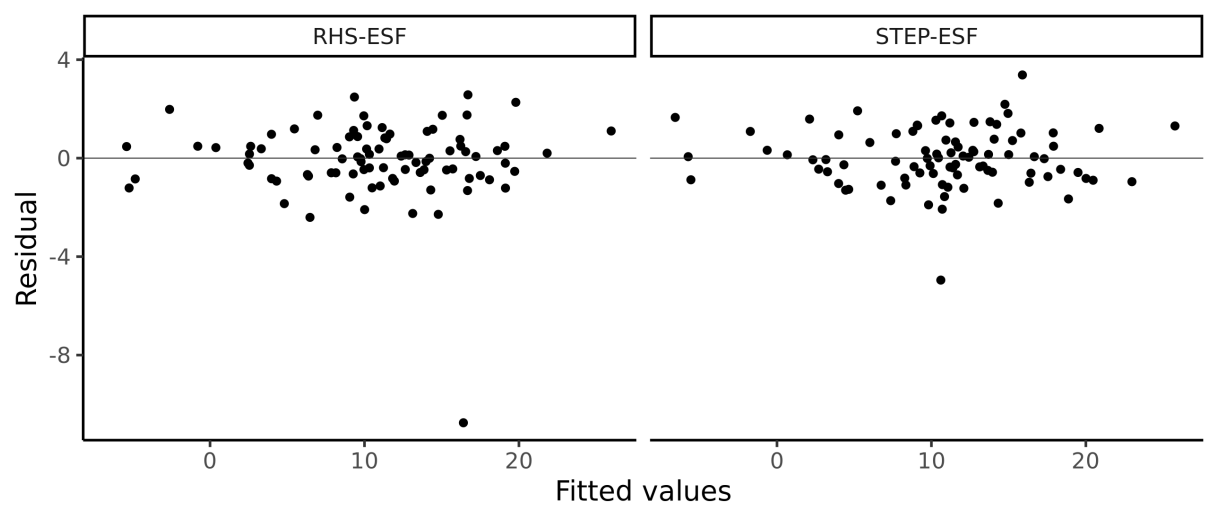

Figure 10: Residuals vs. fitted values for the STEP-ESF and RHS-ESF election models.

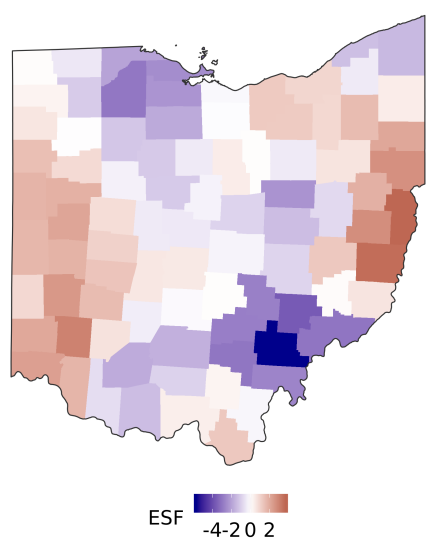

(a) STEP-ESF

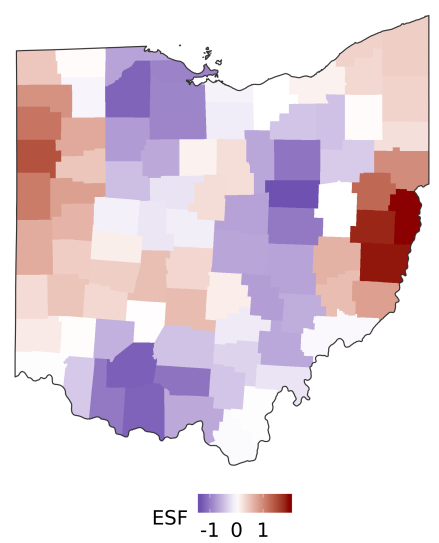

(b) RHS-ESF

Figure 11: Maps of estimated spatial filters for STEP-ESF and RHS-ESF Ohio election models. Note the color schemes are on their own respective scales. 


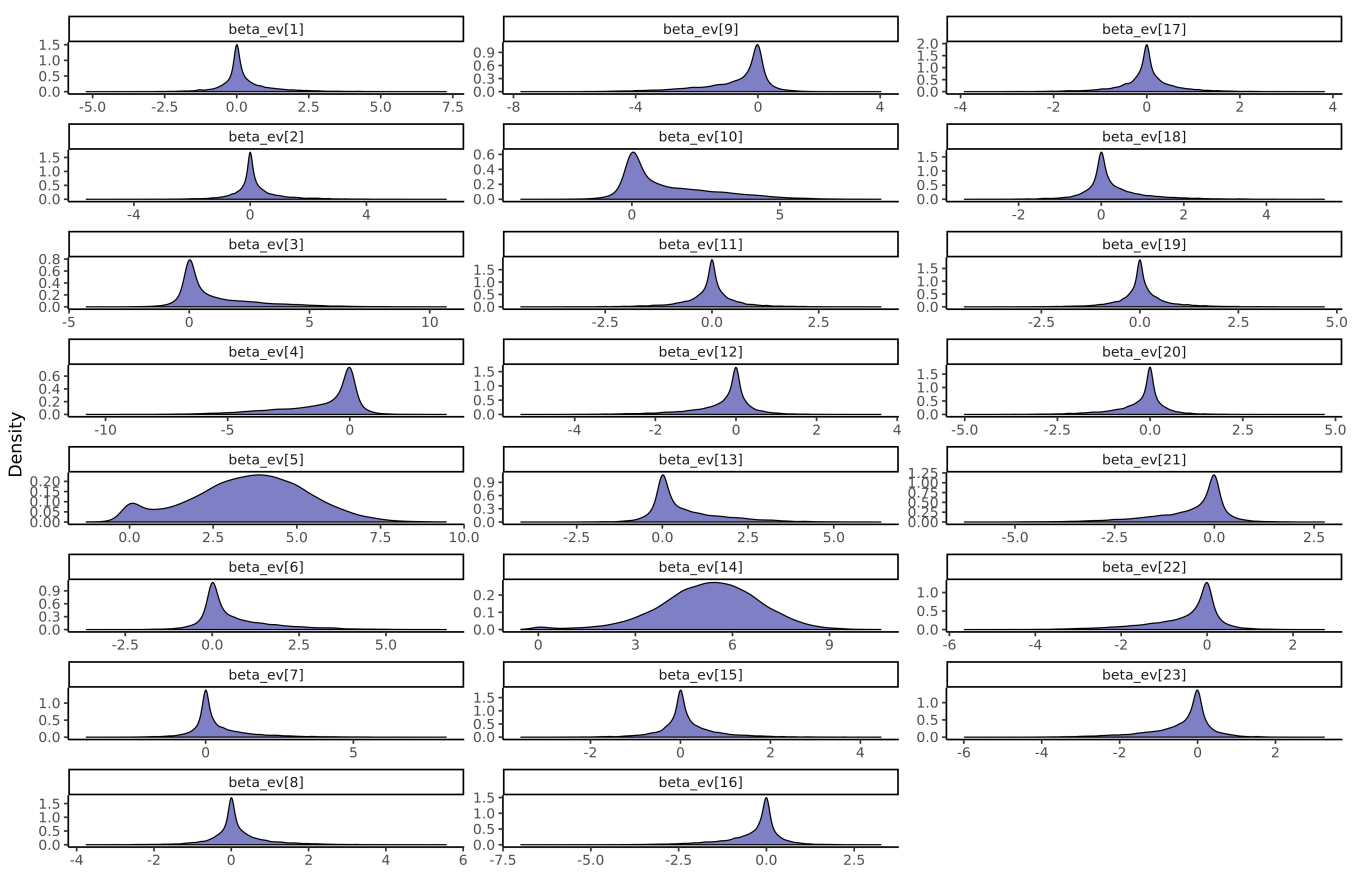

Figure 12: Posterior distributions of eigenvector coefficients in the RHS-ESF election model.
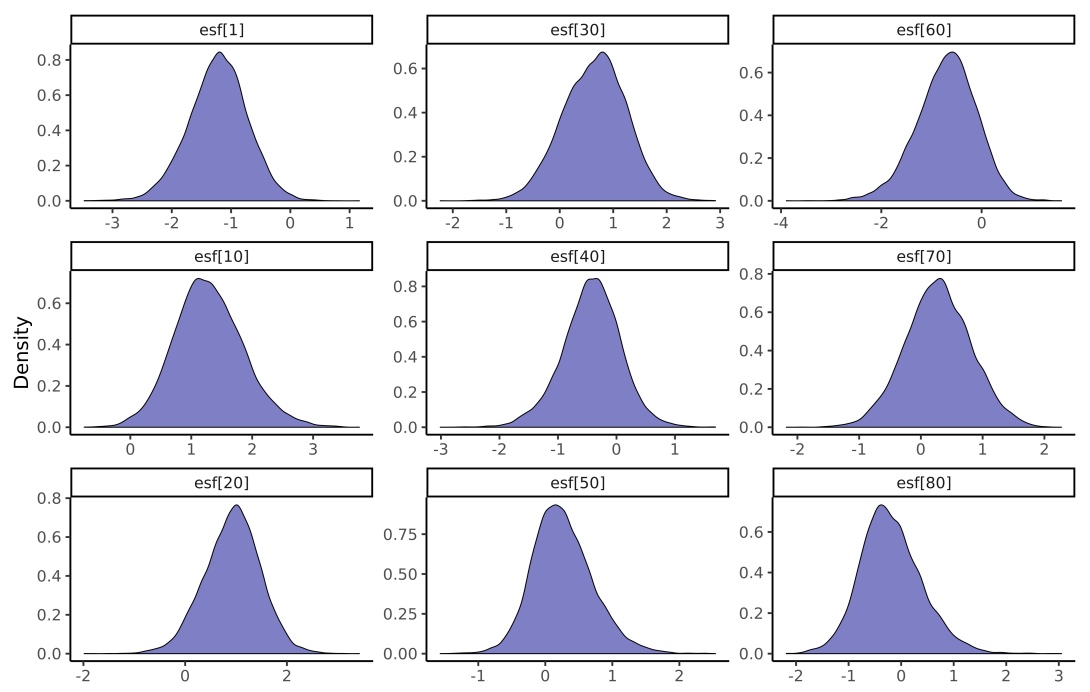

Figure 13: Posterior distribution of the spatial filter $\theta_{i}$ at select observations from the robust RHS-ESF election model. 
Table 2: Estimates from OLS, SAR and STEP-ESF models for the Ohio election data. The dependent variable GOP growth is on its original scale; all covariates have been centered and scaled to have standard deviations of one.

\begin{tabular}{rrrrrrr}
\hline & \multicolumn{2}{c}{ OLS } & \multicolumn{3}{c}{ SAR } & \multicolumn{2}{c}{ STEP-ESF } \\
& Estimate & S.E. & Estimate & S.E. & Estimate & S.E. \\
\hline Intercept & 10.71 & 0.20 & 10.76 & 0.31 & 10.71 & 0.15 \\
w.historic_gop & 0.11 & 0.40 & -0.01 & 0.41 & -0.95 & 0.48 \\
w.log.pop.density & 0.82 & 0.55 & 0.35 & 0.57 & -1.17 & 0.70 \\
w.college_educated & -0.97 & 0.50 & -0.56 & 0.51 & 0.26 & 0.51 \\
w.unemployment & 0.08 & 0.48 & 0.17 & 0.47 & -0.40 & 0.43 \\
historic_gop & -1.37 & 0.41 & -1.33 & 0.35 & -1.90 & 0.35 \\
log(pop.density) & -0.67 & 0.56 & -0.55 & 0.48 & -1.19 & 0.50 \\
white_nonhispanic & 2.24 & 0.47 & 2.39 & 0.41 & 2.66 & 0.42 \\
college_educated & -2.77 & 0.34 & -2.77 & 0.31 & -2.68 & 0.29 \\
unemployment & 1.70 & 0.42 & 1.67 & 0.35 & 1.21 & 0.35 \\
\hline
\end{tabular}

coefficients of independent variables. The paper introduced Piironen and Vehtari's RHS prior as a remedy for this challenge since it accomplishes dimensionality reduction within the rules of probability theory. The RHS-ESF model was then tested in a simulation study designed to ascertain the reliability of parameter estimates under a wide range of SA conditions. The Bayesian ESF model performed as well as the SAR model from which the data was generated while alternative specifications provided overly confident standard errors. These results reinforce the importance of building models that incorporate all sources of uncertainty and also provided insight into the challenge of estimating marginal effects when covariates exhibit strong SA. The results lead to the recommendation that the mean-spatial lag of high-SA covariates be included in the model absent some compelling reason not to. Since the spatial lag term provides useful information about the outcome and corrects the estimated uncertainty of the coefficient, including this term is more than appropriate even when no spatial spillover (causal) effect is theorized to occur. However, the simulation study revealed that highly correlated data $\left(\rho_{x}, \rho_{y}=0.9\right)$ was exceedingly difficult for all of the models to process, leading to useless uncertainty estimates. Lastly, a demonstration analysis of election data showed that the model is highly robust to hyper-prior specifications while Chun et al.'s formula for the expected number of non-zero 
Table 3: Posterior summary of Student's t RHS-ESF regression on growth in the GOP county-level vote share, from the historic average to 2016. GOP growth is on its original scale; covariates have been centered and scaled to have standard deviation one. The intercept represents the expected GOP growth when all covariates are at their mean value. Standard deviation $\sigma$ and degrees of freedom $\nu$ are parameters of the Student's t likelihood function. WAIC: 335.21 , residual $\mathrm{MC}=-0.02$

\begin{tabular}{rcccccc}
\hline & Mean & SD & $2.5 \%$ & $20 \%$ & $80 \%$ & $97.5 \%$ \\
\hline Intercept & 10.82 & 0.14 & 10.54 & 10.70 & 10.93 & 11.09 \\
w.historic_gop & 0.48 & 0.36 & -0.22 & 0.18 & 0.78 & 1.21 \\
w.log.pop.density & 0.48 & 0.49 & -0.52 & 0.08 & 0.89 & 1.41 \\
w.college_educated & -0.74 & 0.43 & -1.60 & -1.10 & -0.38 & 0.12 \\
w.unemployment & 0.40 & 0.38 & -0.36 & 0.08 & 0.72 & 1.15 \\
historic_gop & -2.42 & 0.38 & -3.15 & -2.74 & -2.11 & -1.66 \\
$\log$ (pop.density) & -0.83 & 0.42 & -1.65 & -1.18 & -0.48 & -0.01 \\
white_nonhispanic & 2.92 & 0.38 & 2.18 & 2.61 & 3.23 & 3.66 \\
college_educated & -2.41 & 0.28 & -2.98 & -2.64 & -2.17 & -1.87 \\
unemployment & 1.10 & 0.32 & 0.46 & 0.82 & 1.37 & 1.74 \\
$\sigma$ & 1.08 & 0.16 & 0.79 & 0.95 & 1.20 & 1.41 \\
$\nu$ & 5.29 & 2.68 & 2.16 & 3.28 & 6.85 & 12.14 \\
\hline & & & & & &
\end{tabular}

eigenvector coefficients appears to be a reliable guide for setting the prior degree of sparsity of the model.

Avenues for further research include extending RHS-ESF to Binomial and Poisson models and particularly to disease mapping applications. At the same time, the simulation study could be extended to consider variation across other important parameters such as tessellation size and shape, the signal-to-noise ratio and correlation among covariates, all of which were held constant in this study. While the RHS prior performs exceedingly well, it would be wise to consider alternative sparsity priors as well. The models will be made widely available through a user-friendly $R$ package, geostan . 


\section{Appendix A. Election model}

The Bayesian ESF model in Section 5 can be expressed in probability statements as:

$$
\begin{array}{r}
\boldsymbol{y} \mid \nu, \alpha, \boldsymbol{\gamma}, \boldsymbol{\beta}_{\boldsymbol{x}}, \boldsymbol{\beta}_{\boldsymbol{E}}, \sigma \sim \operatorname{Student}(\nu, \boldsymbol{\mu}, \sigma) \\
\boldsymbol{\mu}=\alpha \mathbf{1}+\boldsymbol{W} \boldsymbol{X} \boldsymbol{\gamma}+\boldsymbol{X} \boldsymbol{\beta}_{\boldsymbol{x}}+\boldsymbol{E} \boldsymbol{\beta}_{\boldsymbol{E}} \\
\alpha \sim \mathrm{N}(0,15) \\
\boldsymbol{\gamma}, \boldsymbol{\beta}_{\boldsymbol{x}} \sim \mathrm{N}(0,6.3) \\
\boldsymbol{\beta}_{\boldsymbol{E}} \sim \operatorname{RHS}\left(\tau_{0}=.43, \nu_{c}=15, s_{c}=15\right) \\
\sigma \sim \mathrm{N}^{+}(0,5) \\
\nu \sim \mathrm{G}(2,0.1)
\end{array}
$$

All covariates are on the unit scale and the standard deviation of GOP growth is 6.3. The priors place low probability on highly implausible values but are largely dominated by the data (with the important exception of $\beta_{E}$ ).

\section{References}

Agnew, J., Shin, M., 2019. Mapping Populism: Taking Politics to the People. Rowman \& Littlefield Publishers.

Bivand, R.S., Pebesma, E.J., Gomez-Rubio, V., Pebesma, E.J., 2008. Applied Spatial Data Analysis with R. Springer.

Borcard, D., Legendre, P., 2002. All-scale spatial analysis of ecological data by means of principal coordinates of neighbour matrices. Ecological modelling 153, 51-68.

Bureau of Labor Statistics, 2016. Local area unemployment statistics. https://www.bls.gov/lau/\#cntyaa.

Carpenter, B., Gelman, A., Hoffman, M.D., Lee, D., Goodrich, B., Betancourt, M., Brubaker, M., Guo, J., Li, P., Riddell, A., 2017. Stan: A probabilistic programming language. Journal of statistical software 76 .

Carvalho, C.M., Polson, N.G., Scott, J.G., 2009. Handling sparsity via the horseshoe, in: Proceedings of the Twelfth International Conference on Artificial Intelligence and Statistics, AISTATS 2009, Clearwater Beach, Florida, USA, April 16-18, 2009, pp. 73-80. 


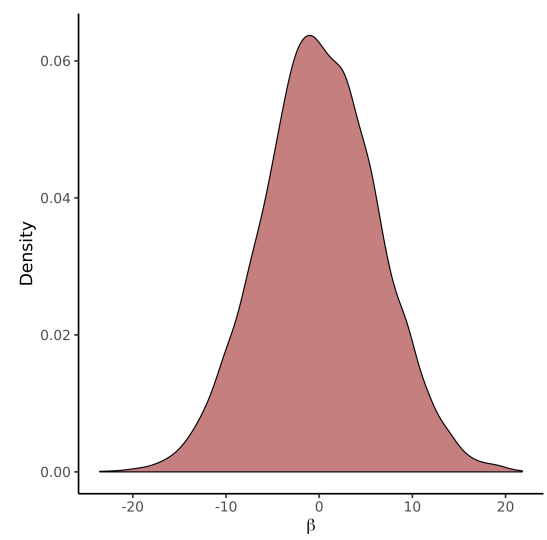

(a) Prior
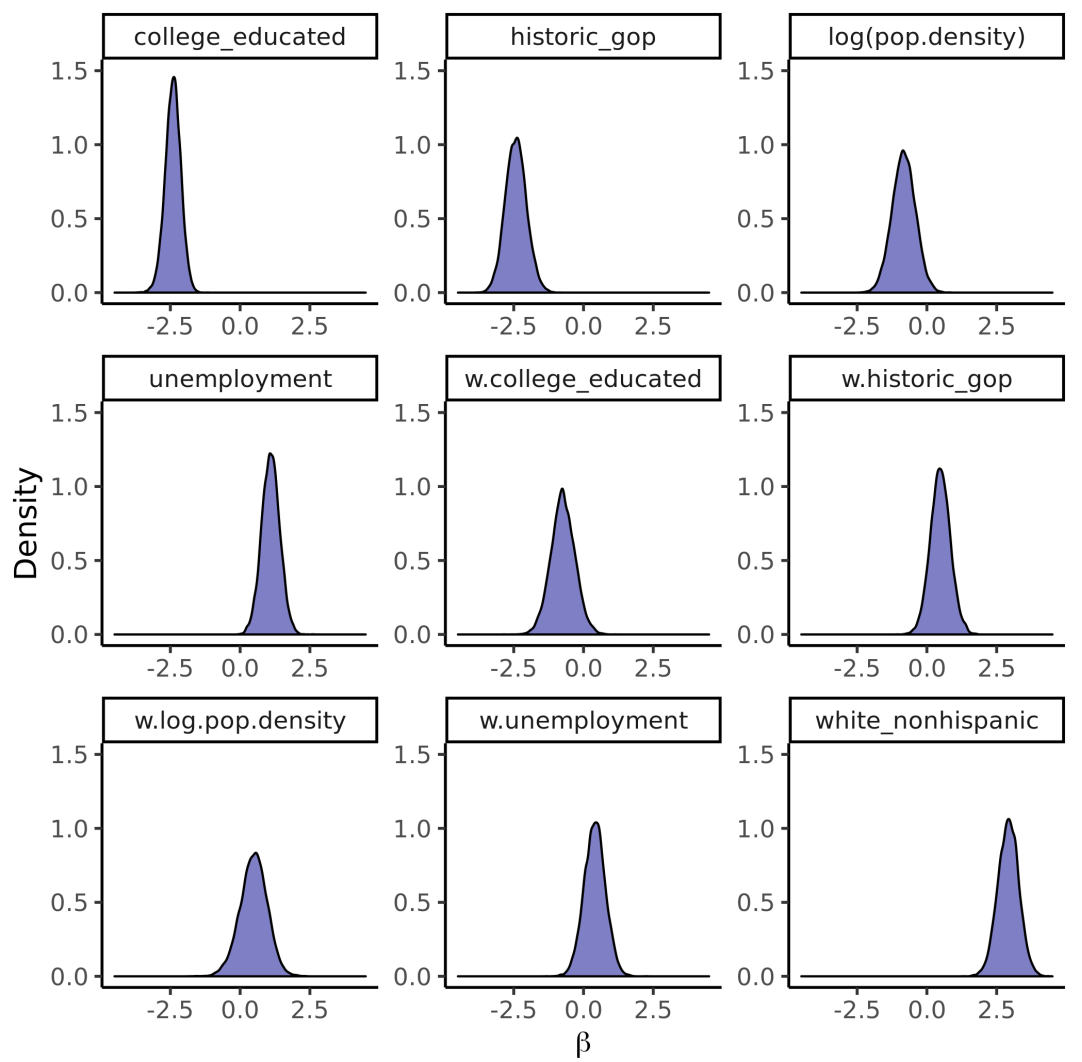

(b) Posterior

Figure A.14: Prior and posterior probability densities for $\beta_{x}$ and $\gamma$. 


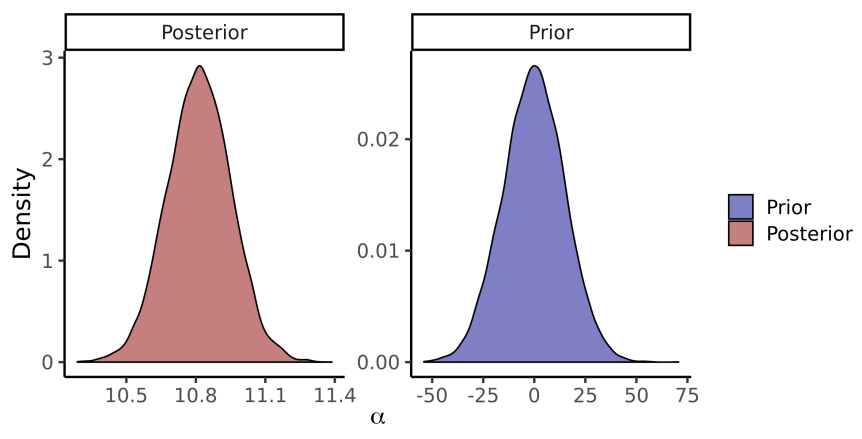

(a) $\alpha$

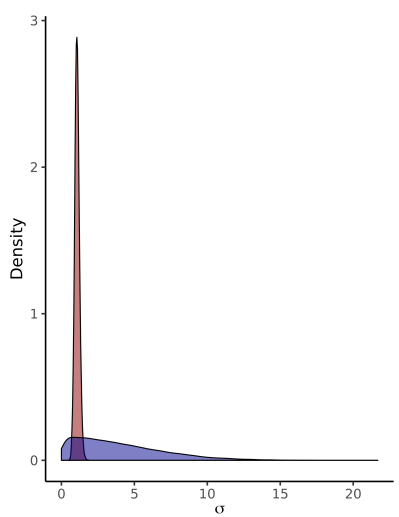

(b) $\sigma$

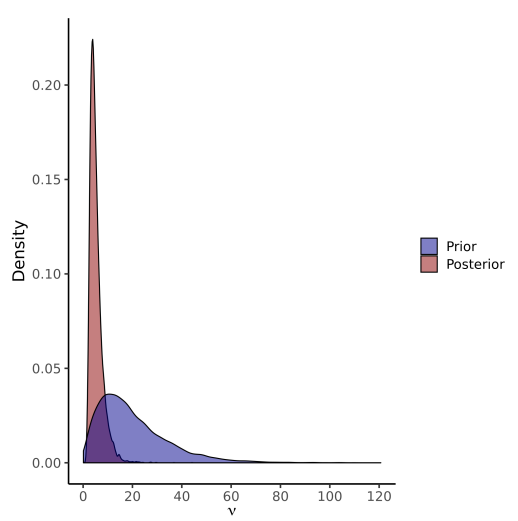

(c) $\nu$

Figure A.15: Prior and posterior probability densities for the intercept $\alpha$, residual standard deviation $\sigma$ and degrees of freedom $\nu$. 
Carvalho, C.M., Polson, N.G., Scott, J.G., 2010. The horseshoe estimator for sparse signals. Biometrika 97, 465-480.

Case, A., Deaton, A., 2020. Deaths of Despair and the Future of Capitalism. Princeton University Press.

Chatfield, C., 1995. Model uncertainty, data mining and statistical inference. Journal of the Royal Statistical Society: Series A (Statistics in Society) $158,419-444$.

Chun, Y., 2008. Modeling network autocorrelation within migration flows by eigenvector spatial filtering. Journal of Geographical Systems 10, 317-344.

Chun, Y., Griffith, D.A., 2013. Spatial statistics and geostatistics: theory and applications for geographic information science and technology. Sage, Los Angeles.

Chun, Y., Griffith, D.A., Lee, M., Sinha, P., 2016. Eigenvector selection with stepwise regression techniques to construct eigenvector spatial filters. Journal of Geographical Systems 18, 67-85.

Cliff, A., Ord, J., 1981. Spatial Processes: Models and Applications. Pion.

Diniz-Filho, J.A.F., de Sant'Ana, C.E.R., Bini, L.M., 1998. An eigenvector method for estimating phylogenetic inertia. Evolution 52, 1247-1262.

Dorn, D., Hanson, G., Majlesi, K., et al., 2016. Importing political polarization? the electoral consequences of rising trade exposure. Technical Report. National Bureau of Economic Research.

Gabry, J., Simpson, D., Vehtari, A., Betancourt, M., Gelman, A., 2019. Visualization in bayesian workflow. Journal of the Royal Statistical Society: Series A (Statistics in Society) 182, 389-402.

Gargallo, P., Miguel, J.A., Salvador, M.J., 2018. Bayesian spatial filtering for hedonic models: An application for the real estate market. Geographical Analysis 50, 247-279.

Gelman, A., Carlin, J.B., Stern, H.S., Dunson, D.B., Vehtari, A., Rubin, D.B., 2014. Bayesian Data Analysis. CRC Press. 3rd edition. 
Geweke, J., 1993. Bayesian treatment of the independent student-t linear model. Journal of applied econometrics 8, S19-S40.

Griffith, D., Chun, Y., 2014. Spatial Autocorrelation and Spatial Filtering, in: Fischer, M.M., Nijkamp, P. (Eds.), Handbook of Regional Science. Springer Berlin Heidelberg, Berlin, Heidelberg, pp. 1477-1507.

Griffith, D., Chun, Y., Li, B., 2019. Spatial Regression Analysis Using Eigenvector Spatial Filtering. Academic Press, London.

Griffith, D.A., 2000. A linear regression solution to the spatial autocorrelation problem. Journal of Geographical Systems 2, 141-156.

Griffith, D.A., 2011. Positive spatial autocorrelation, mixture distributions, and geospatial data histograms, in: IEEE International Conference on Spatial Data Mining and Geographical Knowledge Services, pp. 1-6.

Griffith, D.A., 2012. Spatial statistics: A quantitative geographers perspective. Spatial Statistics 1, 3-15.

Gujarati, D.N., Porter, D.C., Gunasekar, S., 2012. Basic Econometrics. McGraw Hill Education, Chennai.

Haining, R., 1991. Bivariate correlation with spatial data. Geographical Analysis 23, 210-227.

Hodges, J.S., Reich, B.J., 2010. Adding spatially-correlated errors can mess up the fixed effect you love. The American Statistician 64, 325-334.

Hoffman, M.D., Gelman, A., 2014. The no-u-turn sampler: adaptively setting path lengths in hamiltonian monte carlo. Journal of Machine Learning Research 15, 1593-1623.

Hu, L., Griffith, D., Chun, Y., 2018. Space-time statistical insights about geographic variation in lung cancer incidence rates: Florida, usa, 20002011. International journal of environmental research and public health $15,2406$.

Hughes, J., Haran, M., 2013. Dimension reduction and alleviation of confounding for spatial generalized linear mixed models. Journal of the Royal Statistical Society: Series B (Statistical Methodology) 75, 139-159. 
Jaynes, E.T., 2003. Probability theory: the logic of science. Cambridge University Press, Cambridge.

LeSage, J., Pace, R.K., 2009. Introduction to spatial econometrics. CRC Press, Boca Raton.

MIT Election Data and Science Lab, 2018. County Presidential Election Returns 2000-2016. https://doi.org/10.7910/DVN/VOQCHQ.

Monnat, S.M., 2016. Deaths of despair and support for trump in the 2016 presidential election. Pennsylvania State University Department of Agricultural Economics Research Brief 5.

Müller, S., Scealy, J.L., Welsh, A.H., et al., 2013. Model selection in linear mixed models. Statistical Science 28, 135-167.

Murakami, D., 2019. spmoran: Moran's Eigenvector-Based Spatial Regression Models. R package version 0.1.6.2.

Murakami, D., Griffith, D.A., 2015. Random effects specifications in eigenvector spatial filtering: a simulation study. Journal of Geographical Systems 17, 311-331.

Murakami, D., Yoshida, T., Seya, H., Griffith, D.A., Yamagata, Y., 2017. A moran coefficient-based mixed effects approach to investigate spatially varying relationships. Spatial Statistics 19, 68-89.

Oshan, T.M., Fotheringham, A.S., 2018. A comparison of spatially varying regression coefficient estimates using geographically weighted and spatialfilter-based techniques. Geographical Analysis 50, 53-75.

Pace, R.K., LeSage, J.P., Zhu, S., 2013. Interpretation and computation of estimates from regression models using spatial filtering. Spatial Economic Analysis 8, 352-369.

Park, Y.M., Kim, Y., 2014. A spatially filtered multilevel model to account for spatial dependency: application to self-rated health status in south korea. International journal of health geographics 13, 6 .

Piironen, J., Vehtari, A., 2017. Sparsity information and regularization in the horseshoe and other shrinkage priors. Electronic Journal of Statistics $11,5018-5051$. 
Polson, N.G., Scott, J.G., 2010. Shrink globally, act locally: Sparse bayesian regularization and prediction. Bayesian statistics 9, 501-538.

Reich, B.J., Hodges, J.S., Zadnik, V., 2006. Effects of residual smoothing on the posterior of the fixed effects in disease-mapping models. Biometrics 62, 1197-1206.

Seya, H., Murakami, D., Tsutsumi, M., Yamagata, Y., 2015. Application of lasso to the eigenvector selection problem in eigenvector-based spatial filtering. Geographical Analysis 47, 284-299.

Stan Development Team, 2019. RStan: the R interface to Stan. R package version 2.19.2.

"Student" [W.S. Gausset], 1914. The elimination of spurious correlation due to position in time and space. Biometrika 10, 179-180.

Tiefelsdorf, M., Griffith, D.A., 2007. Semiparametric filtering of spatial autocorrelation: the eigenvector approach. Environment and Planning A 39, 1193-1221.

U.S. Census Bureau, 2016. American Community Survey 5-year estimates b01003_001, b15003_001, b15003_022, b03002_003. Accessed from the tidycensus R package, Nov. 6, 2019.

Walker, K., 2019. tidycensus: Load US Census Boundary and Attribute Data as 'tidyverse' and 'sf'-Ready Data Frames. R package version 0.9.2.

Watanabe, S., 2010. Asymptotic equivalence of Bayes cross validation and widely applicable information criterion in singular learning theory. Journal of Machine Learning Research 11, 3571-3594.

Wolf, L.J., Anselin, L., Arribas-Bel, D., 2018. Stochastic efficiency of bayesian markov chain monte carlo in spatial econometric models: An empirical comparison of exact sampling methods. Geographical Analysis 50, 97-119.

Wood, S.N., 2017. Generalized Additive Models: An Introduction with R. CRC Press. 2 edition. 
Yule, G.U., 1926. Why do we sometimes get nonsense-correlations between time-series?-a study in sampling and the nature of time-series. Journal of the royal statistical society $89,1-63$. 